\title{
The Orbital Distribution of Near-Earth Objects Inside Earth's Orbit
}

\author{
by \\ Sarah Greenstreet \\ B.Sc., Western Washington University, 2007 \\ A THESIS SUBMITTED IN PARTIAL FULFILMENT OF \\ THE REQUIREMENTS FOR THE DEGREE OF \\ Master of Science \\ in
}

The Faculty of Graduate Studies

(Astronomy)

The University Of British Columbia

(Vancouver)

September 2011

(c) Sarah Greenstreet 2011 


\section{Abstract}

Canada's Near-Earth Object Surveillance Satellite (NEOSSat), set to launch in early 2012, will search for and track Near-Earth Objects (NEOs), tuning its search to best detect objects with semimajor axis $a<1.0$ AU. In order to construct an optimal pointing strategy for NEOSSat, we needed more detailed information in the $a<1.0$ AU region than the best current model (Bottke et al., 2002) provides. We present here the NEOSSat-1.0 NEO orbital distribution model with larger statistics that permit finer resolution and less uncertainty, especially in the $a<1.0 \mathrm{AU}$ region. We find that Amors $=30.1 \pm 0.8 \%$, Apollos $=63.3 \pm 0.4 \%$, Atens $=5.0 \pm 0.3 \%$, Atiras $(0.718<Q<0.983 \mathrm{AU})=1.38 \pm 0.04 \%$, and Vatiras $(0.307<Q<0.718$ $\mathrm{AU})=0.22 \pm 0.03 \%$ of the steady-state NEO population, where $Q$ is the orbit's aphelion distance. Vatiras are a previously undiscussed NEO population clearly defined in our integrations, whose orbits lie completely interior to that of Venus. Our integrations also uncovered the unexpected production of retrograde orbits from main-belt asteroid sources; this retrograde NEA population makes up $\simeq 0.10 \%$ of the steady-state NEO population. The relative NEO impact rate onto Mercury, Venus, and Earth, as well as the normalized distribution of impact speeds, was calculated from the NEOSSat-1.0 orbital model under the assumption of a steady-state. The new model predicts a slightly higher Mercury impact flux. 


\section{Preface}

- Identification and design of research project: The production of a steady-state NEO orbital distribution model was a project suggested to Sarah Greenstreet by Dr. Brett Gladman. The details of the construction of this model were decided upon by both Sarah Greenstreet and Dr. Gladman.

- Performing the research: Sarah Greenstreet inherited the numerical integrations consisiting of the orbital evolutions at 300-year output intervals of test particles initialized in the asteroid main-belt used to construct the steady-state NEO orbital distribution model which is analyzed in this thesis. In 2008, Dr. Brett Gladman built the initial conditions for the numerical integrations by perturbing those used previously by Bottke et al 2002. The integrations were managed by Henry Ngo from 2008-2009. Sarah Greenstreet finished the numerical integrations from 2009-2010. These numerical integrations can be thought of as a data set inherited by Sarah Greenstreet and the analysis and scientific interpretation of these integrations as well as the construction of the steady-state NEO orbital distribution performed by Sarah Greenstreet with the help of Dr. Gladman are the subject of this thesis.

- Data analysis: The numerical integrations produced a 0.5 Terabtye data set which recorded (in a condensed binary format) the time histories of near-Earth objects from their initial locations until their elimination. Analysis of the numerical integrations dominantly inherited by Sarah Greenstreet was done using pieces of code which extract and analyse the orbital evolution histories of the integrated test particles, construct the residence time distributions of test particles, estimate uncertainties in the residence time distributions, compute the impact speed distributions for Mercury, Venus, and Earth as well as the collision probability distribution for the terrestrial planets. Preliminary versions of some of these codes were written by Henry Ngo. Sarah Greenstreet expanded these codes to extract the analysis presented in 
this thesis. Further information concerning the contributions made by Sarah Greenstreet can be found in Appendix B.

- Manuscript preparation: The majority of this thesis has been submitted to the scientific journal Icarus for publication. This thesis was written entirely by Sarah Greenstreet with the exception of Section 3.1.2 and portions of Section 3.2, which were written by Dr. Brett Gladman. 


\section{Table of Contents}

Abstract $\ldots \ldots \ldots \ldots \ldots \ldots \ldots$ ii

Preface $\ldots \ldots \ldots \ldots \ldots \ldots \ldots \ldots$ iii

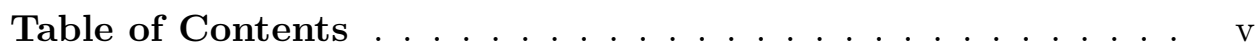

List of Tables $\ldots \ldots \ldots \ldots \ldots \ldots \ldots \ldots$ vii

List of Figures $\ldots \ldots \ldots \ldots \ldots \ldots \ldots \ldots$ viii

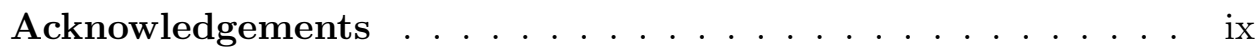

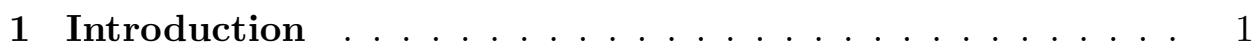

1.1 Motivation .................... 1

1.1.1 Near-Earth asteroid orbital classes . . . . . . . . . 1

1.1.2 Sources of near-Earth objects . . . . . . . . . . 4

1.1.3 NEOSSat: Near-Earth Object Surveillance Satellite . 7

1.1.4 Past work with numerical integrations . . . . . . . 9

2 Methods . . . . . . . . . . . . . . . . . . 12

2.1 Recomputation of the Bottke et al. (2002) integrations . . . 12

2.2 NEOSSat-1.0 NEO orbital model _. . . . . . . . . . . 19

2.2.1 Accuracy of the NEOSSat-1.0 NEO orbital model . . 24

2.3 Caveats . . . . . . . . . . . . . . . . 25

2.3.1 Degeneracy between source regions _ . . . . . . 25

2.3.2 Gravitational effects only included . . . . . . . . . 27

3 Results . . . . . . . . . . . . . . . . . . 28

3.1 New populations discovered within the NEOSSat-1.0 NEO

orbital model . . . . . . . . . . . . . . . . 28

3.1.1 Population of objects decoupled from Venus . . . . 28

3.1 .2 Vulcanoids . . . . . . . . . . . . . . . . 32

3.1 .3 Retrograde NEAs . . . . . . . . . . . . . . 33 
3.2 Impact speeds and rates for Earth, Venus, and Mercury . . . 35

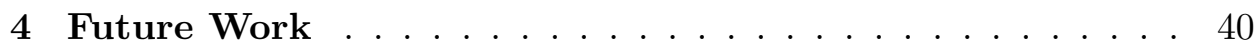

5 Summary and Conclusions ............... 41

Bibliography ..................... 43

Appendices

A Supplementary Information . . . . . . . . . . . 47

B Additional Notes on Co-Authorship . . . . . . . . . . . 52 


\section{List of Tables}

1.1 Glossary of acronyms and symbols . . . . . . . . . . . 2

2.1 Number of integrated test particles . . . . . . . . . . . . . 12

2.2 NEO class percentages and fractional errors . . . . . . . . 15

$2.3 \mathcal{R}_{N E O}(a, e, i)$ from each source region and planet crossing impact rate contributions for NEO classes . . . . . . . . . 21

3.1 Impact flux ratios . . . . . . . . . . . . . . . . . . . . 39 


\section{List of Figures}

1.1 Sample orbits of objects in the four asteroidal NEO classes . 3

1.2 Approximate $(a, i)$ locations of $3: 1$ and $\nu_{6}$ resonances for $e=0$

orbits ...................... 6

2.1 NEO class distinctions and source regions in $a, e$ space . . . 14

$2.2 \mathcal{R}_{N E O}(a, e, i)$ for B02 recomputation . . . . . . . . 16

2.3 Even/odd split of $\mathcal{R}_{N E O}(a, e, i)$ for B02 recomputation . . . 17

$2.4 \mathcal{R}_{N E O}(a, e, i)$ for NEOSSat-1.0 orbital model . . . . . . . . 20

$2.5 \mathcal{R}_{N E O}(a, e, i)$ for $a<1.0$ AU region of NEOSSat-1.0 orbital model . . . . . . . . . . . . . . 23

2.6 Even/odd split of $\mathcal{R}_{N E O}(a, e, i)$ for NEOSSat-1.0 orbital model 26

3.1 First sample Vatira evolution . . . . . . . . . . . . . . 30

3.2 Second sample Vatira evolution . . . . . . . . . . . . . 31

$3.3 \mathcal{R}_{N E O}(a, e, i)$ for inclinations up to $180^{\circ}$ for NEOSSat-1.0

NEO orbital model . . . . . . . . . . . . . . . . . 34

3.4 Impact speed distribution . . . . . . . . . . . . . . 36

A.1 $\mathcal{R}_{\nu_{6}}(a, e, i)$ for NEOSSat-1.0 NEO orbital model . . . . . . . 47

A.2 $\mathcal{R}_{3: 1}(a, e, i)$ for NEOSSat-1.0 NEO orbital model . . . . . . 48

A.3 $\mathcal{R}_{I M C}(a, e, i)$ for NEOSSat-1.0 NEO orbital model . . . . . 49

A.4 $\mathcal{R}_{O M B}(a, e, i)$ for NEOSSat-1.0 NEO orbital model . . . . . 50

A.5 $\mathcal{R}_{J F C}(a, e, i)$ for NEOSSat-1.0 NEO orbital model . . . . 51 


\section{Acknowledgements}

I would like to thank Dr. Brett Gladman, my supervisor, who provided me with support, guidance, and patience as I worked to understand the many concepts of Solar System orbital dynamics associated with this project and planetary sciences in general. I would also like to thank Henry Ngo for the work he put into monitoring the numerical integrations and the initial analysis he performed for this project as well as the comments and insights he provided for portions of this thesis. I also offer my thanks to Jeremy Heyl who acted as second reader for this thesis.

Thanks to Bill McKinnon, Alan Hildebrand, and Bill Bottke for their valuable input and discussions of portions of this thesis. Production of this model was made possible by a Science Support Contract from the Canadian Space Agency. About half of the orbital computations were performed on resources provided by WestGrid and Compute Canada. A portion of the computations were also performed on the GPC supercomputer at the SciNet HPC Consortium. SciNet is funded by: the Canada Foundation for Innovation under the auspices of Compute Canada; the Government of Ontario; Ontario Research Fund - Research Excellence; and the University of Toronto. 


\section{Chapter 1}

\section{Introduction}

\subsection{Motivation}

${ }^{1}$ The Near-Earth Object (NEO) population consists of minor planets (asteroids and de-volatilized comets) with perihelia $q<1.3$ AU. ${ }^{2}$ NEOs originate in the asteroid main-belt and reach near-Earth space via resonances and planetary close encounters. Near-Earth asteroids are scientifically interesting because they were formed 4.5 billion years ago during the formation of the Solar System and can provide valuable information about this process (Hildebrand et al., 2004). Unlike main-belt asteroids, NEOs, especially those with Earth-like orbits, are close enough to be potential targets for future manned and unmanned space probe missions (Abell et al., 2009; Binzel et al., 2010; Hildebrand et al., 2004). Recent missions to have landed on NEOs include NASA's Near-Earth Asteroid Rendevous (NEAR) to 433 Eros (Veverka et al., 2001) and JAXA's Hayabusa to 25143 Itokawa (Yano et al., 2006). The NEO population is the source of impact cratering on the terrestrial planets and a better understanding of the NEO orbital distribution will allow tightening impact chronology (Le Feuvre \& Wieczorek, 2011; Marchi et al., 2009). NEOs with orbits which cross that of the Earth (perihelia less than Earth's aphelion $q<1.017$ AU and aphelion greater than Earth's perihelion $Q>0.983 \mathrm{AU}$ ) can pose a collisional threat to the Earth, thus an understanding of the NEO orbital distribution also serves a non-scientific interest.

\subsubsection{Near-Earth asteroid orbital classes}

According to Kepler's First Law, the Sun acts as a focal point for the elliptical orbits of all Solar System objects. The characteristics which describe

\footnotetext{
${ }^{1} \mathrm{~A}$ version of this chapter has been submitted for publication consideration. Greenstreet, S., Ngo, H., and Gladman, B. (2011) The Orbital Distribution of Near-Earth Objects Inside Earth's Orbit. Icarus.

${ }^{2}$ There is no formal upper limit on semimajor axis for the NEO population although we use $a<4.20 \mathrm{AU}$.
} 
Chapter 1. Introduction

\begin{tabular}{|c|c|}
\hline Acronym/Symbol & Definition \\
\hline $3: 1$ & a main-belt mean-motion resonance \\
\hline$a$ & semimajor axis \\
\hline $\mathrm{AU}$ & astronomical unit \\
\hline Amor & NEO with $1.017<q<1.3 \mathrm{AU}$ \\
\hline Apohele & alternate name for Atira \\
\hline Apollo & NEO with $a>1.0 \mathrm{AU}, q<1.017 \mathrm{AU}$ \\
\hline Aten & NEO with $a<1.0 \mathrm{AU}, Q>0.983 \mathrm{AU}$ \\
\hline Atira & NEO with $0.718<Q<0.983 \mathrm{AU}$ \\
\hline B02 & (Bottke et al., 2002) recomputation \\
\hline$e$ & eccentricity \\
\hline$H$ & absolute magnitude \\
\hline HTC & Halley-type comet \\
\hline$i$ & inclination \\
\hline IEO & NEO with $Q<0.983$ AU \\
\hline IMC & intermediate Mars crossing asteroid \\
\hline JFC & Jupiter-family comet \\
\hline LPC & long-period comet \\
\hline$n$ & mean-motion of an orbiting object \\
\hline NEO & near-Earth object $(q<1.3 \mathrm{AU})$ \\
\hline NEOSSat & Near-Earth Object Surveillance Satellite \\
\hline NEA & near-Earth asteroid \\
\hline $\mathrm{OMB}$ & outer main-belt asteroid \\
\hline$P$ & orbital period \\
\hline$q$ & perihelion distance \\
\hline$Q$ & aphelion distance \\
\hline $\mathcal{R}(a, e, i)$ & residence time probability distribution \\
\hline Vatira & NEO with $0.307<Q<0.718$ AU \\
\hline Vulcanoid & NEO with $Q<0.307 \mathrm{AU}$ \\
\hline$\alpha$ & source region weighting factor for $\mathcal{R}(a, e, i)$ \\
\hline$\mu$ & gravitational parameter, $G\left(M_{\text {Sun }}+m_{\text {object }}\right)$ \\
\hline$\nu_{6}$ & a main-belt secular resonance \\
\hline
\end{tabular}

Table 1.1: Glossary of acronyms and symbols.

these orbits are called orbital elements. The semimajor axis, $a$, measures half the distance of the longest diameter of the orbit, the eccentricity, $e$, describes the deviation of the orbit from a perfect circle, and the inclination, $i$, provides the tilt of the asteroid's orbital plane with respect to the 
Earth-Sun orbital plane. The closest distance to the Sun of an object's orbit (perihelion distance) can be calculated from its $a$ and $e$ via $q=a *(1-e)$. Similarly, an object's furthest distance from the Sun (aphelion distance) can be computed via $Q=a *(1+e)$.

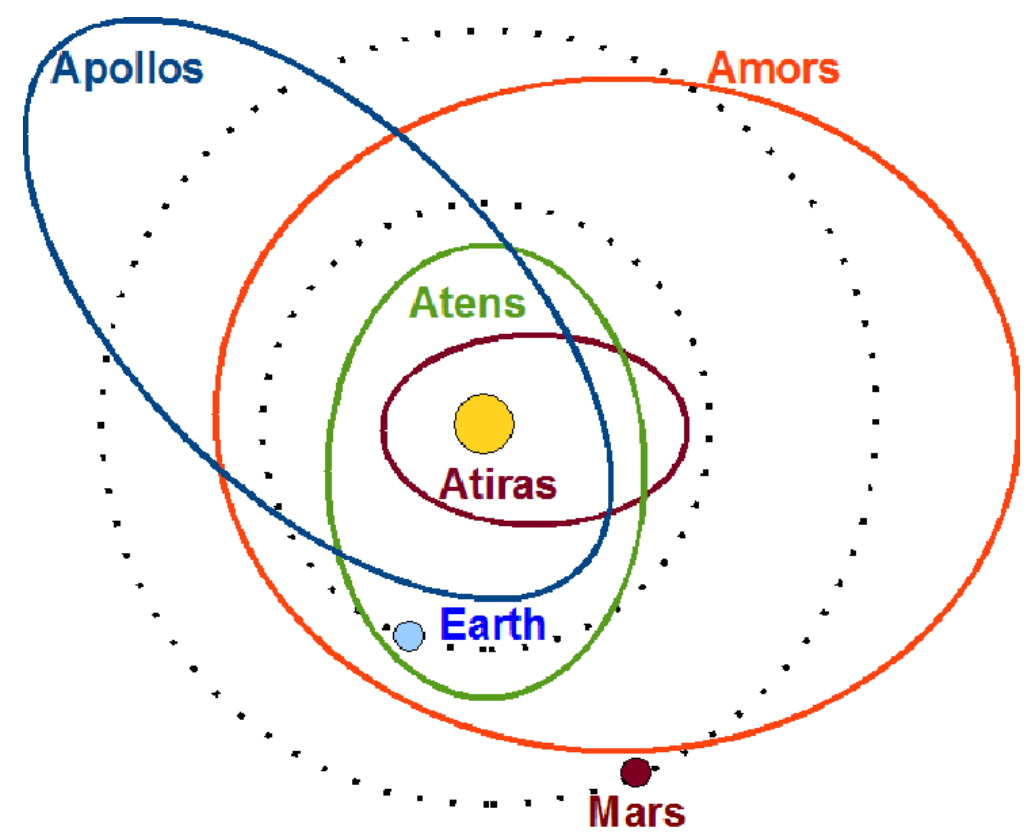

Figure 1.1: Sample schematic orbits of objects in the four traditional asteroidal NEO classes. Apollos and Atens cross the orbit of the Earth while Amors lie entirely exterior to the Earth's orbit and Atiras lie completely interior to the Earth's orbit.

These orbital elements allow the asteroidal component of the NEO population to be divided into the four traditional named classes: Amors $(1.017<$ $q<1.3 \mathrm{AU})$, Apollos $(a>1.0 \mathrm{AU}, q<1.017 \mathrm{AU})$, Atens $(a<1.0 \mathrm{AU}$, $Q>0.983 \mathrm{AU})$, and now Atiras $(0.718<Q<0.983 \mathrm{AU})$, where $Q$ is the orbit's aphelion distance. In recent literature, Atira-class asteroids form part of what has been called Interior-Earth Objects (IEOs) (Michel et al., 2000), because their orbits lie completely interior to Earth's orbit. The name Apoheles, which was suggested by the discoverers of 1998 DK36, is Hawaiian for 'orbit' and was chosen as another name for this NEA class for its similarity to the words 'aphelion' and 'helios'; unfortunately this object became unobservable and is thus not able to be formally named. The NEO 2003 CP20 was discovered by the LINEAR survey and upon being tracked to 
a high-quality orbit was named 163693 Atira (Schmadel, 2009). Following historical precedent, we thus adopt the name Atira for this class of NEO, after its first named member.

Atens and Apollos are Earth-crossing asteroids. Atens have orbits smaller than the Earth's and thus spend much of their time interior to the Earth's orbit while Apollos have orbits larger than the Earth's and spend more time outside the Earth's orbit. These classes make up the potential Earthimpactors. Amors have orbits entirely exterior to the Earth's with perihelia greater than the aphelion of Earth $(q>1.017 \mathrm{AU})$. Atiras, on the other hand, lie on orbits competely interior to the Earth's orbit with aphelion less than the perihelion of Earth $(Q<0.983 \mathrm{AU})$. Atiras are in some sense the symmertric opposite of Amors, having $Q$ touching the Aten population just as Amors have a $q$ boundary on the Apollo interface. Figure 1.1 shows sample schematic orbits of objects in each of the four traditional asteroidal NEO classes. We further expand these into six asteroidal NEO classes (Section 2.1).

\subsubsection{Sources of near-Earth objects}

NEOs originate in the asteroid main-belt and Jupiter-family comet $(P<20$ years) regions (or other cometary reservoirs). There are several paths which can transport objects from either the asteroid main-belt or the Jupiterfamily comet region to near-Earth space. Mean-motion resonances, secular resonances, and planetary close encounters are a few of these paths.

\section{Mean-motion resonances}

Mean-motion resonances located within the asteroid main-belt are an important supply mechanism for NEOs. The mean-motion, $n$, of an object describes the average angular velocity of an object's orbit about the Sun. It is calculated by:

$$
n=\sqrt{\frac{\mu}{a^{3}}}
$$

where $\mu$ is the gravitational parameter and $a$ is the semimajor axis. For objects with $\frac{m_{\text {object }}}{M_{\text {Sun }}}<10^{-11}, \mu=G M_{\text {Sun }}=1.32712440018 \times 10^{20} \mathrm{~m}^{3} \mathrm{~s}^{-2}$. Above this threshold $\mu$ should by calculated as $\mu=G\left(M_{S u n}+m_{\text {object }}\right)$ to maintain the given precision. Mean-motion resonances occur when the asteroidal mean-motion and the planetary mean-motion form a simple fraction. For example, the 2:1 mean-motion resonance with Jupiter occurs when the asteroid orbits the Sun two times for every single orbit of Jupiter, i.e. $2 n_{J}=n_{a}$. Due to the repetition of the positions of the asteroid and planet in a mean-motion resonance, the gravitational interactions between the two 
objects is periodic. This can protect the asteroid from Jupiter's gravitational perturbations which can kick the asteroid out of the inner Solar System and create a stable niche in $(a, e, i)$ space. Other mean-motion resonances are unstable due to intersecting mean-motion resonances located in the asteroid main-belt. The 3:1 mean-motion resonance located near $a \simeq 2.5 \mathrm{AU}$ is one such unstable resonance. The strong e-pumping mechanism within the resonance causes objects located in the resonance to evolve quickly onto Mars-crossing orbits (Wisdom, 1985). Martian close encounters can then kick the object out of the resonance and cause changes in $a$ which place the object in near-Earth space.

\section{Secular resonances}

If the Solar System consisted of only the Sun and one other object, the two orbits would follow the same path around their center of mass indefinitely. However, since there are more than two objects in the Solar System, this is not true. The gravitational perturbations of each object in the Solar System causes the location in $(a, e, i)$ space of the pericenter (the longitude of the pericenter) of each object to precess with time. The precession rate of each planet is a linear combination of the eigenfrequencies associated with the orbital dynamics of the Solar System. A secular resonance occurs when the precession rate of an asteroid's longitude of pericenter matches that of one of the fundamental eigenfrequencies of the Solar System (Michel \& Froeschlé, 1997; Wetherill \& Faulkner, 1981). The $\nu_{6}$ secular resonance, which occurs when the precession rate of the asteroid's longitude of perihelion matches that of the sixth secular frequency of the Solar System, is among the strongest of the secular resonances in the Solar System. The $\nu_{6}$ resonance is located at the inner edge of the asteroid main-belt near $a \simeq 2$ AU and $i<15^{\circ}$. Figure 1.2 shows an $a, i$ plot of the approximate location of the $\nu_{6}$ and $3: 1$ resonances for $e=0$ orbits.

Objects are fed into the $\nu_{6}$ resonance by material in the adjacent mainbelt (area to the right of the $\nu_{6}$ curve in Figure 1.2) as objects random walk in $a$ at roughly constant inclination. Once objects enter the resonance they can quickly have their eccentricities increased to Mars-crossing space where subsequent Martian close encounters can remove the object from the resonance and put it onto a near-Earth orbit.

Lifetimes inside mean-motion and secular resonances are a few hundred thousand years before objects are pushed into the Sun (Gladman et al., 1997). However, planetary close encounters can kick objects either into or out of resonances. If an object leaves the resonance, it can live for tens to hundreds of millions of years random walking in $a$ as it experiences a sequence of planetary close encounters. 


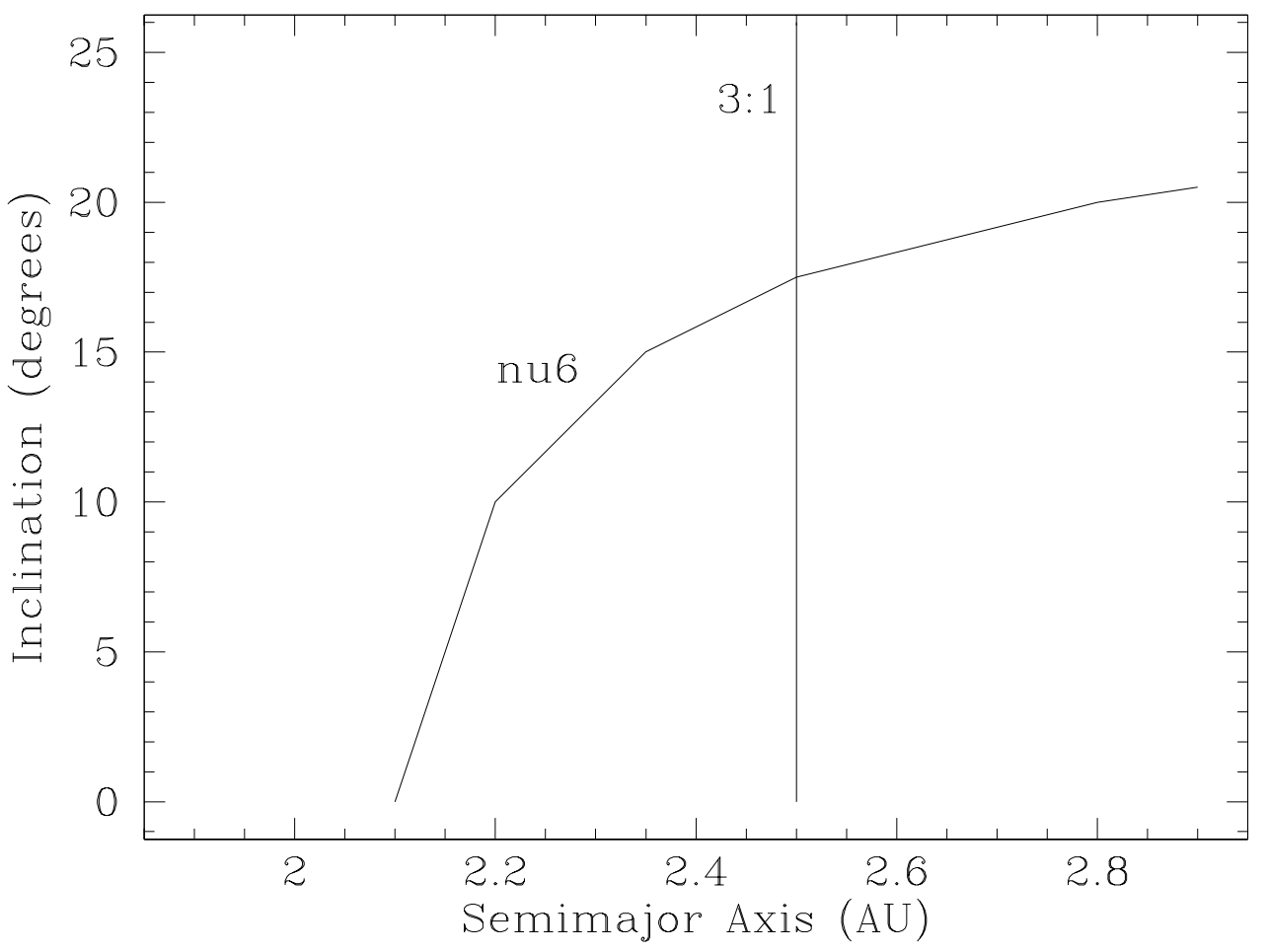

Figure 1.2: Approximate $(a, i)$ locations of the 3:1 mean-motion resonance and $\nu_{6}$ secular resonance for $e=0$ orbits. To the right of the $\nu_{6}$ curve an object has an orbital frequency less than the sixth secular frequency of the Solar System while to the left of the curve an object's orbital frequency is greater than the sixth secular frequency. If an object is locacted on a point along the $\nu_{6}$ curve that object's orbital frequency is equal to the sixth secular frequency of the Solar System and a resonant response occurs. This resonant response can quickly increase the eccentricity of an object to a Sun-grazing orbit within 1 Myr (Farinella et al., 1994) unless a planetary close encounter removes the object from the resonance. A similar resonant response occurs for objects located along the 3:1 mean-motion line shown above.

\section{Intermediate Mars-crossing population}

Asteroids can also escape the main-belt through mean-motion resonances with Mars or three-body mean-motion resonances with the giant planets and reach orbits which cross that of Mars. Martian close encounters can then put objects onto near-Earth orbits. These low-inclination Mars-crossing objects 
with $q>1.3 \mathrm{AU}$ are known (Bottke et al., 2000a) as the Intermediate Mars Crossers (IMC source). This population is located above the $\nu_{6}$ resonance $\left(i>15^{\circ}\right)$ and split by the 3:1 resonance (located near $a \simeq 2.5 \mathrm{AU}$ ) (Bottke et al., 2002). Objects in the IMC population can enter either the $\nu_{6}$ or $3: 1$ resonances and vice versa. The size of this population depends on the eccentricity of Mars which changes on a period of 2 Myr (Bottke et al., 2002). The outer main-belt (OMB) region also has strong mean-motion resonances and three-body resonances which can supply NEOs (Bottke et al., 2002).

\section{Additional potential NEO sources}

Jupiter can scatter Jupiter-family comets (JFCs) onto near-Earth orbits. Jupiter, however, is much more efficient at throwing objects out of the Solar System, so NEOs produced from the JFCs only account for $\simeq 6 \%$ of the total steady-state NEO population (Bottke et al., 2002). Other sources could also feed the NEO population at a minor level. The Hungaria and Phocaea families are both located near the $\nu_{6}$ secular resonance and the 3:1 mean-motion resonance. However, their inclinations are large enough to keep them from entering the $\nu_{6}$ resonance and the planetary close encounters which could push them into near-Earth space are less frequent at high inclinations. Halley-type comets $(20<P<200$ years $)$ and long-period comets $(P>200$ years $)$ can also make their way into near-Earth space as they encounter resonances and experience plantary close encounters. However, because these objects spend most of their time far from the Earth, deter-

mining the size of the populations is difficult. As of 2002 Spacewatch had not discovered any HTCs or LPCs on orbits with $q<1.3$ AU (Bottke et al., 2002). For this reason these sources are currently not considered primary suppliers of the NEO population.

It is a long process to reach near-Earth space from the asteroidal and cometary source regions. It is even more arduous to obtain orbits with $a<1.0 \mathrm{AU}$, making Atens and Atiras the least populous of the NEO classes. This is in part the reason the number of observed Aten and Atira class asteroids is small compared to the number of Amors and Apollos. Atens and Atiras also spend less time at high solar elongations, making them more difficult to observe from ground-based telescopes. These seldom-observed asteroid populations are the focus of NEOSSat's observational goals.

\subsubsection{NEOSSat: Near-Earth Object Surveillance Satellite}

Ground-based telescopes have been quite successful in observing NEOs. In 1989, the Spacewatch program at the Univeristy of Arizona's Lunar and Planetary Laboratory made the first automated discovery of an NEO (1989 
UP) (Gehrels \& Jedicke, 1996). Within the next 7 years, Spacewatch discovered $\approx 75$ NEAs (Gehrels \& Jedicke, 1996). Spacewatch has since increased this number by roughly an order of magnitude. Other surveys, like LINEAR (Stuart, 2001) and Catalina have since discovered thousands ${ }^{3}$ of NEOs. However, ground-based telescopes have several disadvantages when it comes to increasing the census of Atens and Atiras. From the ground, telescopes observing during night hours can only see objects which are located at high enough solar elongations. Although observations at solar elongations less than $60^{\circ}$ are possible from the ground, this is only during a brief time window after or before twilight. Space-based telescopes are unconstrained by the daylight cycle and are not affected by light pollution and weather conditions on the ground. From orbit, their longer baselines allow for parallax distance measurements of objects as well as tracking of fast-moving objects.

Canada's NEOSSat is a joint project between the Canadian Space Agency (CSA) and Defense Research and Development Canada (DRDC) (Hildebrand et al., 2004). The science mission via CSA is to search for and track NEOs, specifically those on orbits with $a<1.0$ AU. NEOSSat is designed similarly to the Microvariability and Oscillations of Stars (MOST) space telescope (Walker et al., 2003). In addition to the advantages listed above of a space-based telescope, NEOSSat features an attached baffle allowing the satellite to look as close as $45^{\circ}$ to the Sun. The currently scheduled launch is set for early 2012 and nominal lifetime is 1 year, although MOST is still operating in 2011 after a 2003 launch (Sarty et al., 2011).

At only $75 \mathrm{~kg}$ NEOSSat is both lightweight and compact with dimensions of $1.0 \mathrm{~m} \times 0.8 \mathrm{~m} \mathrm{x} 0.4 \mathrm{~m}$. It will be in a Sun-synchronous, low-Earth orbit at an altitude of $\approx 650 \mathrm{~km}$. NEOSSat will have a $0.15 \mathrm{~m}$ telescopic mirror with a $0.86^{\circ} \times 0.86^{\circ}$ field of view to detect objects via reflected visible light. A 100 -second exposure time will reach $\approx 20$ th magnitude in the $\mathrm{V}$ band (Hildebrand et al., 2004). It will have two operational modes during science mission operation: a survey mode and a tracking mode. The goal during survey mode is to find and inventory new objects. The software package OpenOrb (Granvik et al., 2009) will be used to determine preliminary orbital parameters and classification of new objects as well as plan recovery observations to determine the orbit of each object. Follow-up will occur during NEOSSat tracking mode and with the help of ground-based telescopes. In order to optimize an efficient pointing strategy for NEOSSat to maximize the number of detections, a model of the NEO orbital distribution with good statistics in the $a<1.0$ AU region is needed.

\footnotetext{
${ }^{3}$ See the JPL NEO Discovery Statistics website: http://neo.jpl.nasa.gov/stats/ .
} 


\subsubsection{Past work with numerical integrations}

Once it was discovered that objects in the main-belt can be fed into the NEO region through resonances, several numerical integrations have been done to model this process and the resulting NEO orbital distribution. During the 1970's, Wetherill developed Monte-Carlo models of collisionally-fragmented objects injected into the $\nu_{6}$ and 3:1 resonances which slowly raised their eccentricities until Mars could gravitationally remove them from the resonance (Wetherill, 1979). Further Martian close encounters caused random walking in $a$ until these objects reached Earth-crossing space. Greenberg \& Chapman (1983) produced similar models of cratering debris from large-body impacts reaching near-Earth space after being injected into resonances. Both models found the path to near-Earth space via resonances took $\approx 1 \mathrm{Myr}$ to reach barely Earth-crossing orbits (Greenberg \& Nolan, 1993) with typical lifetimes of tens of Myr. By the 1990's computers had become powerful enough to perform numerical integrations of statistically significant numbers of objects starting in these resonances. Farinella et al. (1994) showed that on a timescale of $\approx 1 \mathrm{Myr}$, eccentricities of objects injected into orbital resonances can be increased not only to Earth-crossing orbits, but to Sungrazing orbits. This marks the turning point of our modern understanding that main-belt resonances are efficient direct NEA producers. Numerical integrations (Gladman et al., 1997) showed typical dynamical lifetimes of particles placed within many main-belt resonances to be only a few million years, most particles being terminated by becoming Sun grazers or being ejected from the Solar System by Jupiter. Because this dynamical lifetime is much shorter than the age of the Solar System, there must be a stable source resupplying these asteroidal source regions within the main asteroid belt. Collisions (Farinella et al., 1993), semimajor axis drift driven by Yarkovsky (Bottke et al., 2001; Farinella \& Vokrouhlický, 1999), and chaotic dissipation (Carruba et al., 2003; Morbidelli \& Nesvorný, 1999) are possible supply mechanisms for the main-belt orbital resonances.

Morbidelli \& Nesvorný (1999) showed that in addition to the $\nu_{6}$ and 3:1 resonances, Mars-crossing asteroids also efficiently populate the NEO region. This population of Mars-crossing asteroids is produced through chaotic diffusion of objects migrating in $e$, typically living for $\approx 25 \mathrm{Myr}$ (Morbidelli \& Nesvorný, 1999). The IMC population $(q>1.3 \mathrm{AU}, 2.06 \leq a \leq 2.48$ $\mathrm{AU}$ or $2.52 \leq a \leq 2.80 \mathrm{AU}$, and $i \leq 15^{\circ}$ ) is populated by asteroids leaving mean-motion resonances with Mars and three-body mean-motion resonances (Morbidelli \& Nesvorný, 1999) or removed from the 3:1 and $\nu_{6}$ resonances due to Martian close encounters (Bottke et al., 2002). Several mean-motion 
resonances with Jupiter are located in the outer part of the main asteroid belt along with many three-body resonances. These $e$-pumping resonances can also produce chaotic diffusion of outer main-belt (OMB) asteroids onto near-Earth orbits (Morbidelli \& Nesvorný, 1999).

Numerical integrations of the orbits of known short-period comets $(P<$ 200 years) performed by Levison \& Duncan (1994) gave a median lifetime (from the current time) of $\approx 500,000$ years until most objects were either ejected from the Solar System or became Sun grazers. The number of Sun-grazing short-period comets found by these numerical integrations was significantly larger than previous analytical estimates (Levison \& Duncan, 1994). This result pointed to the short-period comet population as a nonnegligible source of NEOs after a large number may lose their cometary aspect due to de-volitalization ${ }^{4}$. Levison \& Duncan (1997) found $\simeq 30 \%$ of the particles evolving out of the Kuiper belt reached orbits with $q<2.5$ $\mathrm{AU}$ at some time during their lives. Some of these have $a<4.2 \mathrm{AU}$ and thus would be NEOs. Because the time spent in the JFC region by the known JFCs is short ( $\approx 0.1$ Myr; (Levison \& Duncan, 1994)), there must be a significant population of objects in the Kuiper belt to supply the known JFC population (Bottke et al., 2002). For this reason the JFC population is also considered a primary NEO source.

The most recent comprehensive set of numerical integrations used to model the NEO population was computed by Bottke et al. (2002). Five source regions were included in these computations: the $\nu_{6}$ secular resonance, the 3:1 mean-motion resonance, the intermediate source Mars crosser (IMC) population, the outer main-belt (OMB) population, and the Jupiterfamily comet (JFC) population. The integrations of particles initialized in the Kuiper Belt (the JFC source region) used by Bottke et al. (2002) were those of Levison \& Duncan (1997). To determine the contribution of each source region to the overall $a<4.2$ AU NEO orbital distribution, Bottke et al. (2002) fit their integrated steady-state for each source to the Spacewatch observations. Their best-fit parameters for the source contributions were $\alpha_{\nu_{6}}=0.37 \pm 0.08, \alpha_{I M C}=0.27 \pm 0.03, \alpha_{3: 1}=0.20 \pm 0.08$, $\alpha_{O M B}=0.10 \pm 0.01$, and $\alpha_{J F C}=0.06 \pm 0.04$ which can be interpreted as the fraction of the steady-state NEO population from each source. Note that in a given sub-region of orbital parameter space these fractions can vary widely; for example, more than $37 \%$ of the Atens come from the $\nu_{6}$ since the JFC and OMB sources contribute no Atens. This model also broke

\footnotetext{
${ }^{4}$ We adopt the terminology that a $q<1.3$ AU object with coma is a comet and not an $\mathrm{NEO}$, otherwise the object is termed a near-Earth comet (NEC) and not considered here.
} 
down the resulting predicted population into each NEO class ${ }^{5}$, with Amors constituting $31 \pm 1 \%$, Apollos $61 \pm 1 \%$, Atens $6 \pm 1 \%$, and IEOs $2 \pm 0 \%$ of the NEO population, where we assume the final IEO uncertainty was not zero, but rather $<0.5 \%$. We will further break the IEO population into 3 subgroups in Section 2.1 with Atiras as the most populous of these groups.

Though the dominant population regions (Amors and Apollos) of the NEO orbital distribution are well represented in the Bottke et al. (2002) model, we concluded that the uncertainty in the $a<1.0$ AU region was too large to plan an optimal pointing strategy for NEOSSat to discover and track Atens and Atiras. We also had concern that the exclusion of Mercury from their integrations could have caused the $a<1.0$ AU populations to be distributed differently than in their orbital model. For these reasons we were motivated to compute a new steady-state NEO orbital distribution, with more test particles and greater integrator accuracy.

In this paper, we first present a recomputation of the Bottke et al. (2002) integrations in order to prove reproducibility and understand the effect of small-number statistics present in the model. We then present a new set of numerical integrations to model the steady-state NEO orbital distribution with better statistics in the $a<1.0$ AU region. The NEOSSat-1.0 NEO orbital model consists of approximately six times as many test particles as the Bottke et al. (2002) model. We show the uncertainty in the $a<1.0$ AU NEO populations of the NEOSSat-1.0 model are significantly improved. A description of two previously undiscussed populations seen in the NEOSSat1.0 model can be found in Section 3.1. Section 3.2 discusses the relative NEO impact rate onto Mercury, Venus, and Earth along with the normalized distribution of impact speeds computed for the NEOSSat-1.0 model.

\footnotetext{
${ }^{5}$ Bottke et al. (2002) normalized their population fractions only to the $Q>0.983 \mathrm{AU}$ region. We have included the near-Sun populations and renormalized these fractions.
} 


\section{Chapter 2}

\section{Methods}

\subsection{Recomputation of the Bottke et al. (2002) integrations}

${ }^{6}$ Due to recent increases in computational power, we present here new numerical integrations similar to those of Bottke et al. (2002) with several improvements. Our first set of integrations was a simple recomputation of the Bottke et al. (2002) integrations with similar initial conditions, using only a smaller timestep, in order to prove reproducibility. In fact, we obtained the precise initial conditions used by the previous integrations. Although chaotic dynamics would have in any case generated rapidly-diverging trajectories from the previous study, we decided to slightly perturb the initial orbits. To generate new initial conditions for our integrations, the value of

\begin{tabular}{|c|c|c||c|}
\hline $\begin{array}{c}\text { Source } \\
\text { region }\end{array}$ & $\begin{array}{c}\text { Bottke et al. } \\
\mathbf{( 2 0 0 2 )}\end{array}$ & $\begin{array}{c}\text { B02 } \\
\text { Recomputation }\end{array}$ & $\begin{array}{c}\text { NEOSSat-1.0 } \\
\text { Model }\end{array}$ \\
\hline$\nu_{6}$ & 3519 & 3000 & 27000 \\
$3: 1$ & 2354 & 987 & 19740 \\
IMC & 2977 & 5568 & 11136 \\
OMB & 1964 & 1964 & 3928 \\
\hline Total & 10814 & 11519 & 61804 \\
\hline
\end{tabular}

Table 2.1: Number of test particles integrated for each source region for the Bottke et al. (2002) model, our B02 recomputation, and the NEOSSat-1.0 model.

the mean anomaly for particles starting in the $\nu_{6}$ resonance, IMC population, and OMB population were re-randomized from that used by Bottke et al. (2002). For particles in the 3:1 mean-motion resonance, both the longitude of perihelion and mean anomaly were changed, but in a way that

\footnotetext{
${ }^{6} \mathrm{~A}$ version of this chapter has been submitted for publication consideration. Greenstreet, S., Ngo, H., and Gladman, B. (2011) The Orbital Distribution of Near-Earth Objects Inside Earth's Orbit. Icarus.
} 
preserved the value of the resonant argument, keeping the particles in the resonance.

Table 2.1 shows the number of particles integrated for each of the four asteroidal source regions for the Bottke et al. (2002) model, our Bottke et al. (2002) (B02) recomputation, and the NEOSSat-1.0 NEO orbital model (discussed in the next Section). The total number of test particles integrated by Bottke et al. (2002) and for our B02 recomputation are comparable, though we integrated less than half as many particles initialized in the 3:1 resonance and twice as many in the IMC source region. For the initial orbital elements of particles originating in each source region see Bottke et al. (2002) Section 2 and their Figure 1. These source regions are also shown schematically in Figure 2.1.

The N-body code SWIFT-RMVS4 was used to perform this computation $^{7}$ with a default timestep of $\approx 6$ hours. This tiny timestep allowed highspeed encounters with Earth and Venus to be correctly resolved. A record of the orbital elements of all test particles was logged every 1000 years. The gravitational perturbations of Venus-Neptune were included. Mercury was not present in these integrations for the purpose of the recomputation. Once a test particle reached the NEO region it was tracked until it either struck the Sun, struck a planet, travelled outside 19 AU from the Sun, or the final integration time was reached. Test particles were monitored for $150 \mathrm{Myr}$ in each asteroidal source region.

Construction of the steady-state NEO orbital distribution was the primary goal. This is expressed via a grid of $a, e, i$ cells throughout the inner Solar System covering $a<4.2 \mathrm{AU}, e<1.0$, and $i<90^{\circ}$ with volume 0.05 AU $\times 0.02 \times 2.00^{\circ}$. This is 12.5 times the resolution used by Bottke et al. (2002). The cumulative time spent by all particles in each cell was normalized to the total time spent by all particles in all cells of the $q<1.3 \mathrm{AU}$ and $a<4.2 \mathrm{AU}$ NEO region. This determines the steady-state NEO orbital distribution supplied by each source region. These residence time probability distributions, $\mathcal{R}_{x}(a, e, i)$, can be interpreted as the steady-state fraction of NEOs from each source region $x$ in that cell. Each source region is then weighted by the best-fit parameters ${ }^{8}$ of Bottke et al. (2002) to make the

\footnotetext{
${ }^{7}$ The rmvs4 variant of the SWIFT package (Levison 2008, private communication) maintains the planetary positions to be the same regardless of timestep changes induced by test-particle proximity.

${ }^{8} \alpha_{\nu_{6}}=0.37, \alpha_{I M C}=0.27, \alpha_{3: 1}=0.20, \alpha_{O M B}=0.10, \alpha_{J F C}=0.06$
} 


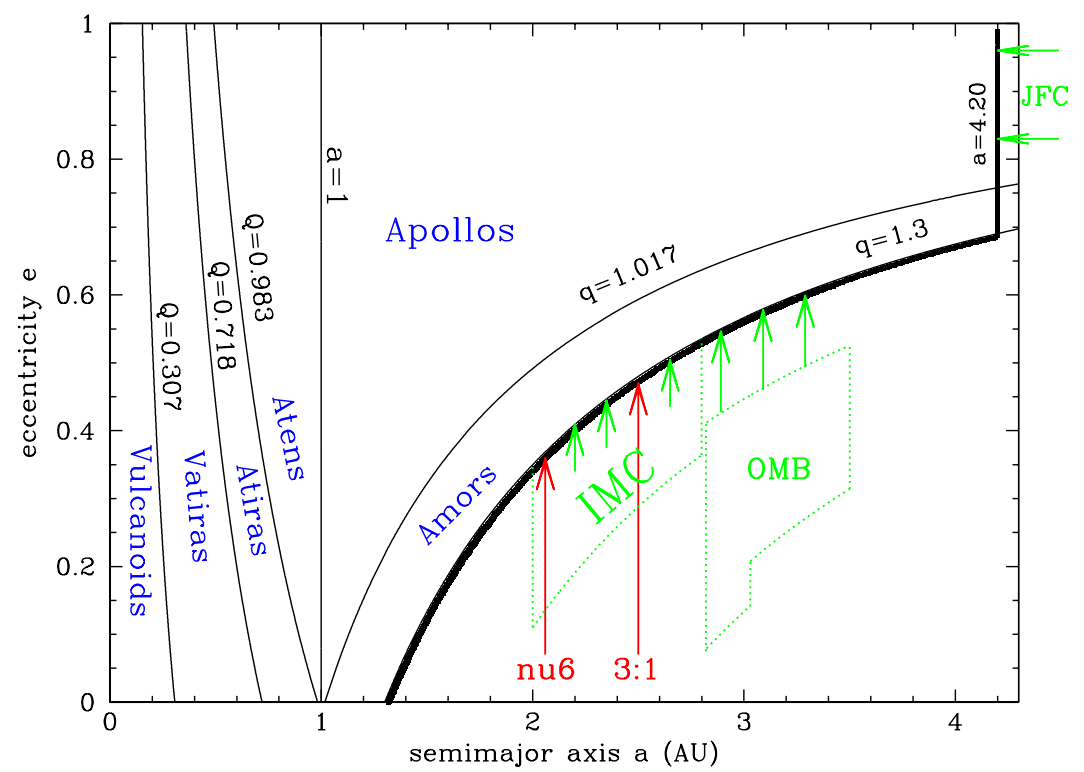

Figure 2.1: NEO class distinctions and source regions in a,e space. We restrict the NEO population to orbits with $q<1.3 \mathrm{AU}$ and $a<4.2 \mathrm{AU}$. Amors (1.017 $<q<1.3 \mathrm{AU})$, Apollos $(a>1.0 \mathrm{AU}, q<1.017 \mathrm{AU})$, Atens $(a<1.0 \mathrm{AU}, Q>0.983 \mathrm{AU})$, Atiras $(0.718<Q<0.983 \mathrm{AU})$, Vatiras $(0.307<Q<0.718 \mathrm{AU})$, and Vulcanoids $(Q<0.307 \mathrm{AU})$ are the six NEO classes (blue) we adopt in this paper. The $\nu_{6}$ secular resonance (red), 3:1 mean-motion resonance (red), intermediate Mars crossers (IMC) (green), and the outer main-belt (OMB) (green) population constitute the asteroidal source regions and the Jupiter family comets (JFCs) (green) are the cometary source region used in the B02 recomputation and the NEOSSat-1.0 NEO orbital integrations. 
combined residence time distribution, $\mathcal{R}_{N E O}(a, e, i)$, of the NEO region.

$\mathcal{R}_{N E O}(a, e, i)=\alpha_{\nu_{6}} \mathcal{R}_{\nu_{6}}+\alpha_{I M C} \mathcal{R}_{I M C}+\alpha_{3: 1} \mathcal{R}_{3: 1}+\alpha_{O M B} \mathcal{R}_{O M B}+\alpha_{J F C} \mathcal{R}_{J F C}$

Though no particles of cometary origin were integrated for this recomputation, $\mathcal{R}_{J F C}(a, e, i)$ from Bottke et al. (2002) was available and used.

\begin{tabular}{|c|c|c|c|c|c|}
\hline & $\begin{array}{c}\text { Bottke et al. } \\
(\mathbf{2 0 0 2})\end{array}$ & \multicolumn{2}{|c|}{$\begin{array}{c}\text { B02 } \\
\text { Recomputation }\end{array}$} & \multicolumn{2}{c|}{$\begin{array}{c}\text { NEOSSat-1.0 } \\
\text { Model }\end{array}$} \\
\hline NEO & $\begin{array}{c}\text { Best } \\
\text { Estimate } \\
\mathbf{( \% )}\end{array}$ & $\begin{array}{c}\text { Best } \\
\text { Estimate } \\
\mathbf{( \% )}\end{array}$ & $\begin{array}{c}\text { Fractional } \\
\text { Error }\end{array}$ & $\begin{array}{c}\text { Best } \\
\text { Estimate } \\
\mathbf{( \% )}\end{array}$ & $\begin{array}{c}\text { Fractional } \\
\text { Error }\end{array}$ \\
\hline Amor & $31 \pm 1$ & $32.0 \pm 0.1$ & 0.003 & $30.1 \pm 0.8$ & 0.025 \\
Apollo & $61 \pm 1$ & $57.7 \pm 3.0$ & 0.053 & $63.3 \pm 0.4$ & 0.007 \\
Aten & $6 \pm 1$ & $5.5 \pm 1.5$ & 0.28 & $5.0 \pm 0.3$ & 0.05 \\
Atira & -- & $3.9 \pm 1.2$ & 0.31 & $1.38 \pm 0.04$ & 0.03 \\
Vatira & -- & $0.9 \pm 0.4$ & 0.47 & $0.22 \pm 0.03$ & 0.15 \\
Vulcanoid & -- & $0.0 \pm 0.0$ & -- & $0.0 \pm 0.0$ & -- \\
\hline IEO & $2 \pm 0$ & $4.8 \pm 1.6$ & 0.34 & $1.6 \pm 0.1$ & 0.05 \\
\hline
\end{tabular}

Table 2.2: NEO class percentages and fractional errors for both our B02 recomputation and the NEOSSat-1.0 NEO model. An Atira has $0.718<$ $Q<0.983$ AU while a Vatira is a Venus-decoupled object with $0.307<$ $Q<0.718$ AU. We define Vulcanoids as objects with $Q<0.307$ AU. The uncertainties in our percent class estimates and the resulting fractional errors are computed using the even/odd splits of the residence time distributions. For comparison, the NEO class percentages computed by Bottke et al. (2002) and renormalized to $100 \%$ are also given. Bottke et al. (2002) combine all $Q<0.983$ AU objects into the IEO population. The IEO class percentages for our B02 recomputation and the NEOSSat-1.0 NEO model are simply the sum of the Atira, Vatira, and Vulcanoid class percentages. The best estimates for our B02 recomputation and the NEOSSat-1.0 model agree (within our estimated errors) with the Bottke et al. (2002) NEO class best estimates.

Figure 2.2 shows two projections of $\mathcal{R}_{N E O}(a, e, i)$ for our B02 recomputation. In order to estimate how much of this residence time is due to small-number stastistics fluctuations, some of which are due to single particles, two residence time distributions were computed by splitting the evenand odd-numbered particle contributions of the asteroidal source regions. The test particles were numbered via the sequential incrementation of the 

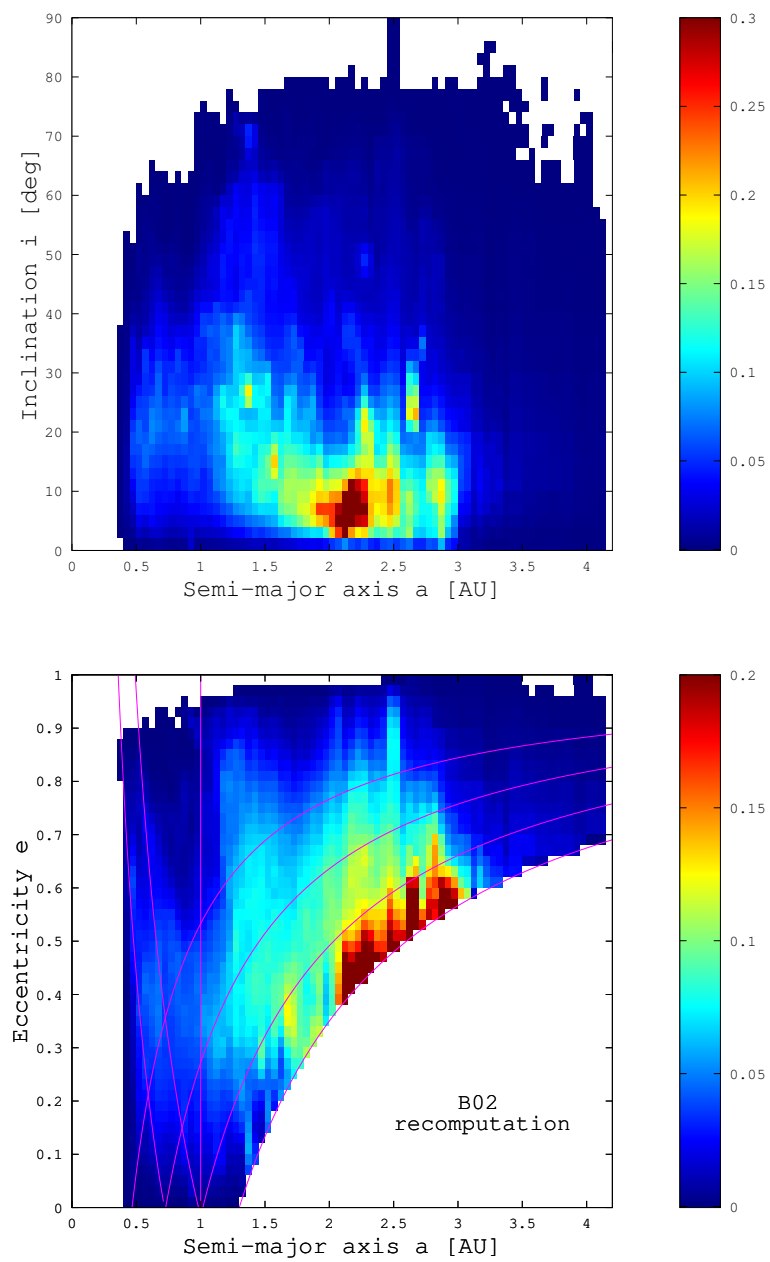

Figure 2.2: Residence time probability distribution, $\mathcal{R}_{N E O}(a, e, i)$, from our recomputation of the Bottke et al. (2002) integrations using a smaller timestep. To monitor the orbital evolution of each particle, a grid of $a$, $e, i$ cells was placed throughout the inner Solar System from $a<4.2 \mathrm{AU}$, $e<1.0$, and $i<90^{\circ}$ with volume $0.05 \mathrm{AU} \times 0.02 \times 2.00^{\circ}$ (Bottke et al., 2002). To create the $a, e$ plot the $i$ bins are summed and the $e$ bins are summed to create the $a, i$ plot. The color scheme represents the percentage of the steady-state NEO population contained in each bin. Red colors represent cells where there is a high probability of particles spending their time. The curved lines divide the NEO region into Amor, Apollo, Aten, and Atira populations as well as indicate Venus and Mercury crossing orbits. 

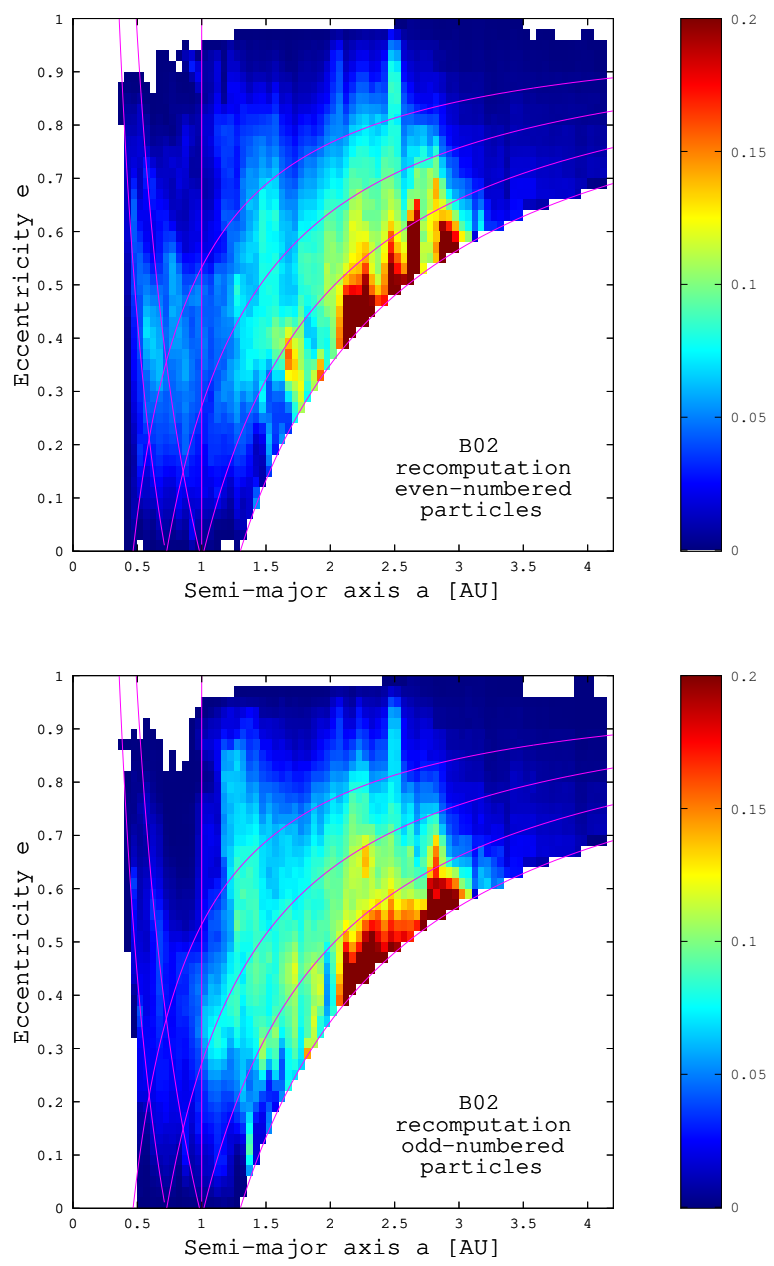

Figure 2.3: In order to determine how much of the residence time probability distribution displayed in Figure 2.2 is due to small-number stastistics fluctuations, some of which are due to single particles, the residence time was computed by splitting the even- and odd-numbered particle contributions of the asteroidal source regions. Two projections of the resulting $\mathcal{R}_{N E O}(a, e, i)$ are shown here. The even-numbered particle contribution is shown in the top plot while the odd-numbered particle contribution is shown in the bottom plot. The discrepancies between these two plots show the potential importance of small-number statistics in our B02 recomputation. Particularly, the $a<1.0 \mathrm{AU}$ regions show large discrepencies in the NEO population. The fractions of NEOs in each class vary by a factor of 2-3 between the even and odd particle splits. 
initial conditions for each source region; thus, splitting the even- and oddnumbered particle contributions gives two comparable NEO distributions with very similar initial conditions. The resulting residence time probability distributions are shown in Figure 2.3. The discrepancies between these two plots give estimates of the importance of small-number statistics in our B02 recomputation. Particularly, the $a<1.0$ AU regions of Figure 2.3 show large discrepencies in the NEO populations, varying by factors of 2-3.

Table 2.2 shows that the best estimates for the NEO class fractions for our B02 recomputation agree (within our estimated errors) with the conclusions of Bottke et al. (2002). Note that Bottke et al. (2002) combined all $Q<0.983$ AU objects into the IEO population (Table 2.2) while we split this region into three subpopulations: Atiras $(0.718<Q<0.983 \mathrm{AU})$, Vatiras $(0.307<Q<0.718 \mathrm{AU})$ which are objects decoupled from Venus, and Vulcanoids which we require to have orbits with $Q<0.307$ AU (the perihelion of Mercury). For a graphical representation, Figure 2.1 shows the NEO class boundaries we adopt in this paper. The IEO class percentage given for the B02 recomputation and the NEOSSat-1.0 model are simply the sum of the Atira, Vatira, and Vulcanoid class percentages. Vatiras and Vulcanoids are discussed in greater detail in Section 3.1.

The best estimates given in Table 2.2 are an average of the even/odd split NEO class fractions. The percent errors given for the best estimates are half of the difference in the NEO class fractions for the even/odd splits of $\mathcal{R}_{N E O}(a, e, i)^{9}$. The fractional errors given in Table 2.2 are percent errors for each NEO class divided by the fractional best estimate ${ }^{10}$. The Bottke et al. (2002) uncertainties were originally computed by varying their best-fit parameters for the source regions within their estimated uncertainties and recomputing the NEO class fractions (Bottke 2011, private communication), but this neglects the uncertainties due to small-number statistics in the integrations themselves. The fractional errors of $0.3-0.5$ for the $a<1.0$ AU region show the potential importance of small-number statistics in the B02 recomputation. Since this is the region of phase space NEOSSat will be focusing its observations, we were motivated to compute a new set of numerical integrations to model the steady-state distribution of the NEO population with better statistics in the $a<1.0$ AU region.

\footnotetext{
${ }^{9}$ For example, the even split of the B02 recomputation gives Atens $=7.0 \%$ of the NEO population while the odd split gives Atens $=4.0 \%$. Therefore, the percent error is $(7.0 \%-$ $4.0 \%) / 2$ or $1.5 \%$.

${ }^{10}$ For example, the $1.5 \%$ error given for the Aten estimate of the B02 recomputation divided by $5.5 \%$ (the best estimate) is 0.27 , so the relative fractional uncertainty is about 1 part in 4 .
} 


\subsection{NEOSSat-1.0 NEO orbital model}

For the numerical integrations of the NEOSSat-1.0 orbital model, we used SWIFT-RMVS4 with a base timestep of $\approx 4$ hours. Note that the code adaptively reduces the timestep down to $\approx 10$ minutes in deep planetary encounters. Due to computational limitations a decade ago, Bottke et al. (2002) were unable to consider including the gravitational effects of Mercury in their integrations. The 4-hour timestep allows us to resolve even the highest-speed Mercury encounters in our computation. The inclusion of Mercury is important in our efforts to better model the $a<1.0$ AU NEO population. We include the gravitational perturbations of Mercury-Saturn. Only point-mass Newtonian gravity is included. The orbital elements of all particles were recorded every 300 years. Particles were tracked from their starting points in one of the source regions until they reached one of the following sinks: hit the Sun, hit a planet, reached transuranian space $(Q>19 \mathrm{AU})$, or the final integration time was reached. Almost no particles remained with $q<1.3 \mathrm{AU}$ at the end of the integrations.

The same perturbed initial condition algorithm for each asteroidal source region used in the B02 recomputation was used, but roughly six times as many particles were tracked (Table 2.1). 27000 particles starting in the $\nu_{6}$ secular resonance were followed for $200 \mathrm{Myr}$. 19740 particles starting in the 3:1 mean-motion resonance were followed for 200 Myr. 11136 particles starting in the IMC population were followed for $150 \mathrm{Myr}$ and 3928 particles starting in the OMB population were followed for $100 \mathrm{Myr}$. The variation in total integration time for the 4 source regions reflects the need to integrate until $>99 \%$ of the $q<1.3 \mathrm{AU}$ particles were eliminated. Altogether, these integrations took more than 300 core years of computation to complete using the fastest cores available in 2009. The output of the entire integration set is available upon request.

The same bins $\left(a<4.2 \mathrm{AU}, e<1.0\right.$, and $i<90^{\circ}$ with volume 0.05 $\left.\mathrm{AU} \times 0.02 \times 2.00^{\circ}\right)$ were used to express $\mathcal{R}_{N E O}(a, e, i)$ for the NEOSSat1.0 model as our B02 recomputation. We continue to use $\mathcal{R}_{J F C}(a, e, i)$ computed by Bottke et al. (2002) to compute $\mathcal{R}_{N E O}(a, e, i)$. Figure 2.4 shows the combined residence time distribution of the five source regions, again using the best-fit weightings of Bottke et al. (2002).

The particle evolutions do not show surprises compared to previous integrations, except for the rare outcomes mentioned in Section 3.1. Largely, particles which enter the $q<1.3$ AU NEO region scatter gravitationally off the planets and migrate around in $(a, e, i)$ space. Most are removed quickly when they encounter a strong resonance (usually the $\nu_{6}$ or $3: 1$ ) and end in 

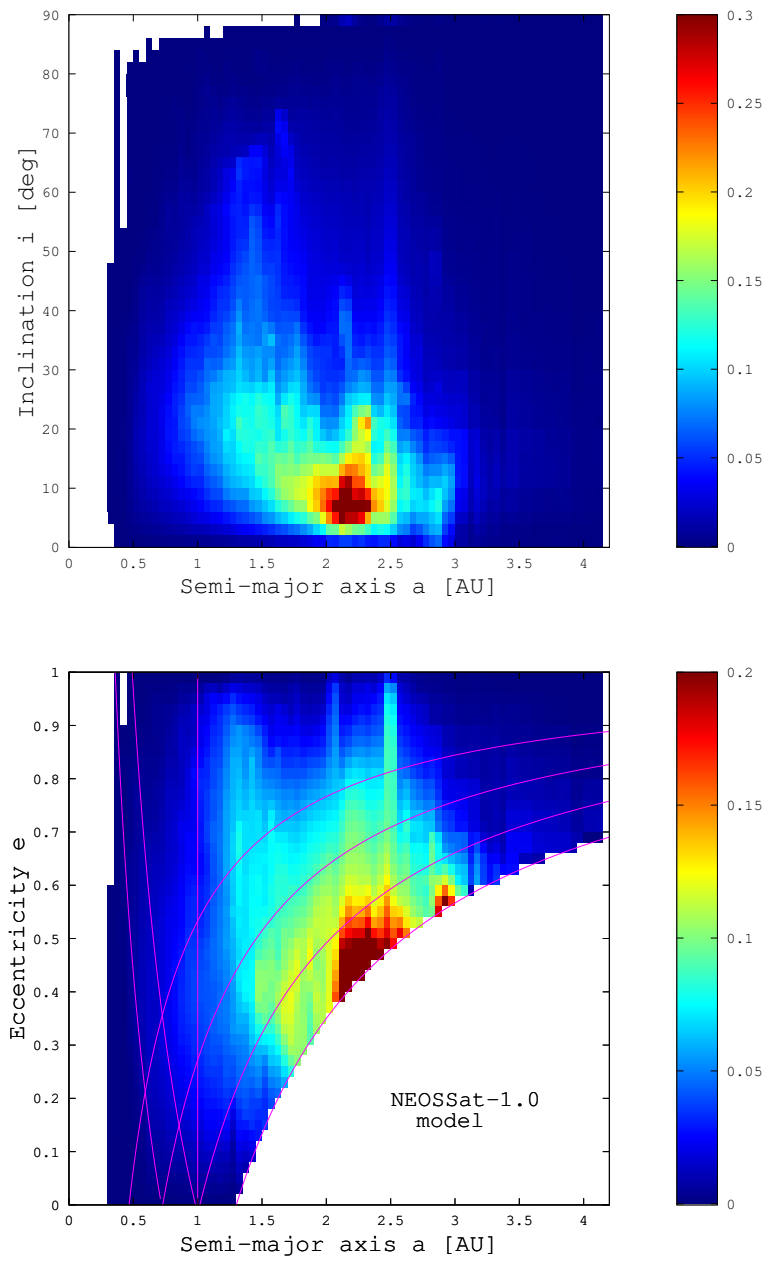

Figure 2.4: Residence time probability distribution, $\mathcal{R}_{N E O}(a, e, i)$, for the NEOSSat-1.0 NEO orbital model. This figure is constructed the same as Figure 2.2. 
a Sun-grazing state. These paths to high-e via the $\nu_{6}$ and 3:1 resonances can be seen in Figure 2.4 at $a \simeq 2 \mathrm{AU}$ and $a \simeq 2.5 \mathrm{AU}$, respectively. The median eccentricity of objects in the $a>2 \mathrm{AU}$ NEO region is $e \simeq 0.5$ and rises slightly at lower $a$ (Figure 2.4). In particular, this produces a concentration of Mercury-crossing Apollos at $a \simeq 1.2 \mathrm{AU}$. The median inclination for NEOs with $a>2 \mathrm{AU}$ is $\simeq 15^{\circ}$ with slight deficits at $a \simeq 2 \mathrm{AU}$ and $a \simeq 2.5$ AU where the $\nu_{6}$ and 3:1 resonances do not allow objects to increase much in inclination before being pushed into the Sun. The median inclination rises to $>25^{\circ}$ at $a<1.5 \mathrm{AU}$.

\begin{tabular}{|c|c|c|c|c|c|}
\hline & Vatira & Atira & Aten & Apollo & Amor \\
\hline \% $\nu_{6}$ & $81.3 \pm 7.5$ & $61.5 \pm 0.5$ & $52.1 \pm 1.8$ & $38.5 \pm 0.2$ & $30.0 \pm 0.2$ \\
\% 3:1 & $9.6 \pm 4.3$ & $15.3 \pm 0.2$ & $20.7 \pm 2.5$ & $21.3 \pm 0.5$ & $17.5 \pm 0.5$ \\
\% IMC & $9.1 \pm 3.3$ & $23.2 \pm 0.7$ & $27.2 \pm 0.7$ & $24.5 \pm 0.2$ & $32.4 \pm 0.8$ \\
\% OMB & 0 & 0 & 0 & $9.8 \pm 0.9$ & $12.7 \pm 1.6$ \\
\% JFC & 0 & 0 & 0 & 6.0 & 7.3 \\
\hline \% Earth Impact & -- & -- & $20.3 \pm 0.9$ & $79.7 \pm 0.9$ & -- \\
\% Venus Impact & -- & $16.5 \pm 0.1$ & $19.8 \pm 0.6$ & $63.7 \pm 0.5$ & -- \\
\% Mercury Impact & $3.2 \pm 0.9$ & $13.1 \pm 0.2$ & $26.4 \pm 0.2$ & $57.3 \pm 0.9$ & -- \\
\hline
\end{tabular}

Table 2.3: The top portion gives the fraction of $\mathcal{R}_{N E O}(a, e, i)$ from each source region for each NEO class. Note the increasing relative importance of the $\nu_{6}$ source for NEO classes nearer the Sun. The bottom portion gives the planet crossing impact rate contributions from NEO classes (Sec. 3.2). This can be interpreted as Atens contribute $20.3 \%$ of the Earth crossing impact rate and Apollos contribute $79.7 \%$, etc. The uncertainties for both portions are computed using the even/odd particle splits of the residence time distribution, expect for the JFC population where an uncertainty estimate is unavailable.

The NEOSSat-1.0 model predicts Amors to be $30.0 \pm 0.8 \%$ of the steadystate NEO population and Apollos to be $63.3 \pm 0.4 \%$ of the population (Table 2.2). These best estimates agree (within our estimated uncertainties) with those of Bottke et al. (2002). In addition, the NEOSSat-1.0 model also allows the computation of the fraction of $\mathcal{R}_{N E O}(a, e, i)$ from each source region for each NEO class (top portion of Table 2.3). For example, $21 \%$ of the Apollos come from the 3:1 resonance. Note that $\mathcal{R}_{N E O}(a, e, i)$ gives the relative NEO class fractions, not the absolute NEO population. To order of magnitude, given there are $\sim 1000$ NEOs with $H<18$ (Bottke et al., 2002; Stuart, 2001), these estimates imply there should be of order 300 Amors and 600 Apollos of this size at any time, with more at smaller diameters. 
For $H<21$, there are $~ 20,000$ NEOs, implying of order 6,000 Amors and 13,000 Apollos exist of this size at any time.

The H-magnitude (absolute magnitude) of a Solar System object is defined to be the apparent magnitude that object would have if it were located in an equilateral triangle $1 \mathrm{AU}$ from the Sun and the Earth (observer) and at zero phase angle. The phase angle refers to the angle between the incident and reflected light of an observed object. The phase angle ranges from $0^{\circ}$ to $180^{\circ}$, where a $0^{\circ}$ phase angle refers to a fully illuminated object. H-magnitude can be used to estimate the diameter of an object. The relationship between $\mathrm{H}$-magnitude and diameter involves the albedo (reflectivity) of an object. The albedo refers to the fraction of incident light reflected from the surface of an object. An albedo of 0 means an object reflects no light and a value of 1 means an objects reflects all light incident upon it. The typical albedo range of NEOs is 0.05-0.25. The diameter of an object can be calculated from the H-magnitude and albedo by:

$$
D=\frac{1329 k m}{\sqrt{A}} 10^{-0.2 H}
$$

An NEO with $\mathrm{H} \sim 18$ has a diameter range of roughly $0.67 \mathrm{~km}$ to $1.5 \mathrm{~km}$ for the estimated albedo range.

NEOSSat's observations will be optimized to maximize $a<1.0$ AU detections, particularly seeking Atira-class asteroids. Figure 2.5 shows two projections of $\mathcal{R}_{N E O}(a, e, i)$ rescaled for $a<1.0$ AU from the NEOSSat1.0 orbital model. The median eccentricity for Atens, Atiras, and Vatiras is $\simeq 0.4$. Figure 2.4 shows that the median inclination climbs steadily from $\simeq 10^{\circ}$ at $a \simeq 2 \mathrm{AU}$ to $\simeq 30^{\circ}$ at $a \simeq 1.2 \mathrm{AU}$ and drops to $\simeq 25^{\circ}$ for Atens, Atiras, and Vatiras (Figure 2.5). The median inclination uncertainty is about a degree, based on the inclination bin size of $\mathcal{R}_{N E O}(a, e, i)$ and the even/odd particle split.

Figure 2.5 shows clear population drop-offs between Atens and Atiras as well as between Atiras and Vatiras. The typical path of NEAs moving from the main-belt to $a<1.0 \mathrm{AU}$ orbits occurs via planetary close encounters and resonances. Once objects enter Earth-crossing space via resonances they often approximately begin following lines of constant Tisserand parameter roughly parallel to $q \simeq 1 \mathrm{AU}$ until planetary close encounters put them onto $a<1.0$ AU orbits (Michel et al., 2000). If NEAs succeed in reaching the Aten and Atira regions, their dynamical lifetimes from that instant then increases due to their orbital distance from the resonances which can push them into the Sun (Gladman et al., 2000). Protection mechanisms, such as 

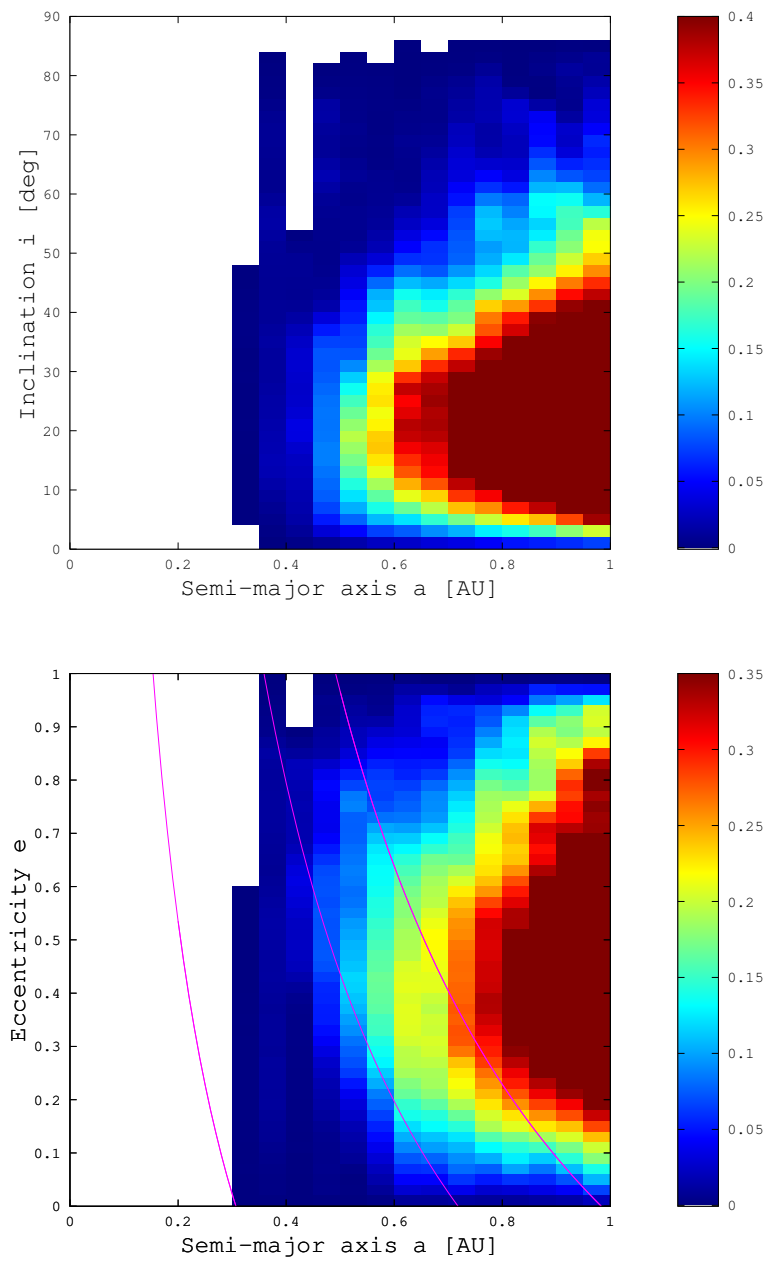

Figure 2.5: Same as Figure 2.4 except displaying only the $a<1.0$ AU region of the NEOSSat-1.0 model. The right edge of the plots mark the Aten/Apollo boundary. Clear population drop-offs are visible at $Q=0.983$ $\mathrm{AU}$ (aphelion at Earth, to the right of which are Atens) and $Q=0.718 \mathrm{AU}$ (aphelion at Venus, to the left of which are Vatiras, with Atiras occupying the region between the curved lines). 
Kozai, also increase their lifetimes while on $a<1.0$ AU orbits (Michel \& Thomas, 1996; Michel et al., 2000).

Table 2.3 makes it clear that the NEO classes have different contributions from the five source regions. The OMB and JFC sources supply essentially no objects to the $a<1 \mathrm{AU}$ region. It is also apparent that the inner belt $\left(\nu_{6}\right.$ and IMC) sources become progressively more dominant for NEO classes with lower and lower semimajor axis. For example, the $\nu_{6}$ source contributes nearly two thirds of the Atira asteroids but only about one third of the Apollos. Because the source regions are likely feeding asteroids of different spectral types into the NEO population, a difference in average colors (for example) between Atens and Apollos does not necessarily imply an evolutionary effect with time.

The NEOSSat-1.0 orbital model predicts Atens to be $5.0 \pm 0.3 \%$ of the steady-state NEO population, Atiras to be $1.38 \pm 0.04 \%$, and Vatiras to be $0.22 \pm 0.03 \%$ of the population (Table 2.2). These estimates are smaller than those computed for the B02 recomputation, but agree within the larger estimated errors of the latter. The uncertainty for these predictions are significantly lower for the NEOSSat-1.0 model. In addition to computing source region fractions for each NEO class, the fraction of $\mathcal{R}_{N E O}(a, e, i)$ from each source region can also be done at an individual cell level, to determine the likelihood that an NEO came from a given source region. For example, the cell containing NEO 163693 Atira $(a=0.741 \mathrm{AU}, e=0.322$, $\left.i=25.6^{\circ}\right)$ has $55 \pm 6 \%$ of $\mathcal{R}_{N E O}(a, e, i)$ coming from the $\nu_{6}$ resonance, $22 \pm 6 \%$ from the $3: 1$ resonance, $24 \pm 6 \%$ from the IMC population and $0 \%$ from the OMB and JFC populations. This can be interepreted as Atira having $\mathrm{a} \simeq 55 \%$ chance of originating in the $\nu_{6}$ resonance, etc. To determine the variation in this estimate, the mean fraction of $\mathcal{R}_{N E O}(a, e, i)$ coming from each source was found by moving up and down one cell each in $a, e$, and $i$. The uncertainties given are the standard deviation of this variation. This source region mapper for the NEOSSat-1.0 model is available upon request. To convert from relative NEO class estimates to absolute values, given there are $\sim 1000$ NEOs with $H<18$, these estimates imply there should be of order 50 Atens, 15 Atiras, and 2 Vatiras of this size at any time, with more at smaller diameters. For $H<21$, there are $\sim 20,000$ NEOs, implying of order 1,000 Atens, 275 Atiras, and 40 Vatiras exist of this size at any time.

\subsubsection{Accuracy of the NEOSSat-1.0 NEO orbital model}

Our usage of roughly six times as many particles as the Bottke et al. (2002) integrations allows finer resolution. To graphically illustrate the reduced 
importance of small-number statistics, we split the NEOSSat-1.0 orbital integrations into even- and odd-numbered particle contributions in Figure 2.6. The discrepancies between these two plots are clearly now much smaller, especially in the $a<1.0 \mathrm{AU}$ region, compared to the $\mathcal{R}_{N E O}(a, e, i)$ projections shown in Figure 2.3, as would be expected given the small fractional errors.

Estimates (Table 2.2) for the steady-state NEO class percentages for both the NEOSSat-1.0 NEO orbital model and our B02 recomputation are all within our estimated uncertainties for both. The new NEO class contributions are within twice our estimated uncertainties of the original Bottke et al. (2002) values, but since we are using their same weighting factors this may not be surprising. The fractional error estimates for the Apollo population is now a factor of eight smaller. We did not refit our integrations to the Spacewatch data because our analysis above shows that in the Apollo and Amor regions, which dominate the Spacewatch detections, the NEOSSat-1.0 and Bottke et al. (2002) models are in agreement, and thus the weightings will not change. It would be more beneficial to fit our integrations to a new NEO survey, such as the NEOWISE data (Mainzer et al., 2011). The fractional errors make it clear we succeeded in decreasing the uncertainty in the fractional populations of the $a<1.0 \mathrm{AU}$ objects by a factor of more than five. The number of Atens and Atiras for our B02 recomputation varied by a factor of 2-3 between the even- and odd-numbered particle splits. However, the fractional errors of these two populations for the NEOSSat1.0 model are now less than 0.10. Since this is the region NEOSSat will be focusing its observations, we are confident that the NEOSSat-1.0 orbital model gives a better representation of the NEA steady-state distribution for the Aten and Atira regions than previous models. The variation in the population of Mercury-crossing Apollos at $a \simeq 1.2$ AU in the NEOSSat-1.0 model (Figure 2.6) is much smaller than before and thus this feature is a robust property of the $\mathrm{NEO}$ distribution.

\subsection{Caveats}

Two possible issues with the NEOSSat-1.0 NEO orbital model which may affect the interpretation of the model include degeneracy between source regions and the exclusion of non-gravitational effects in the integrations.

\subsubsection{Degeneracy between source regions}

One possible issue with the weightings found by Bottke et al. (2002) and used for the NEOSSat-1.0 NEO model integrations is the degeneracy between the 

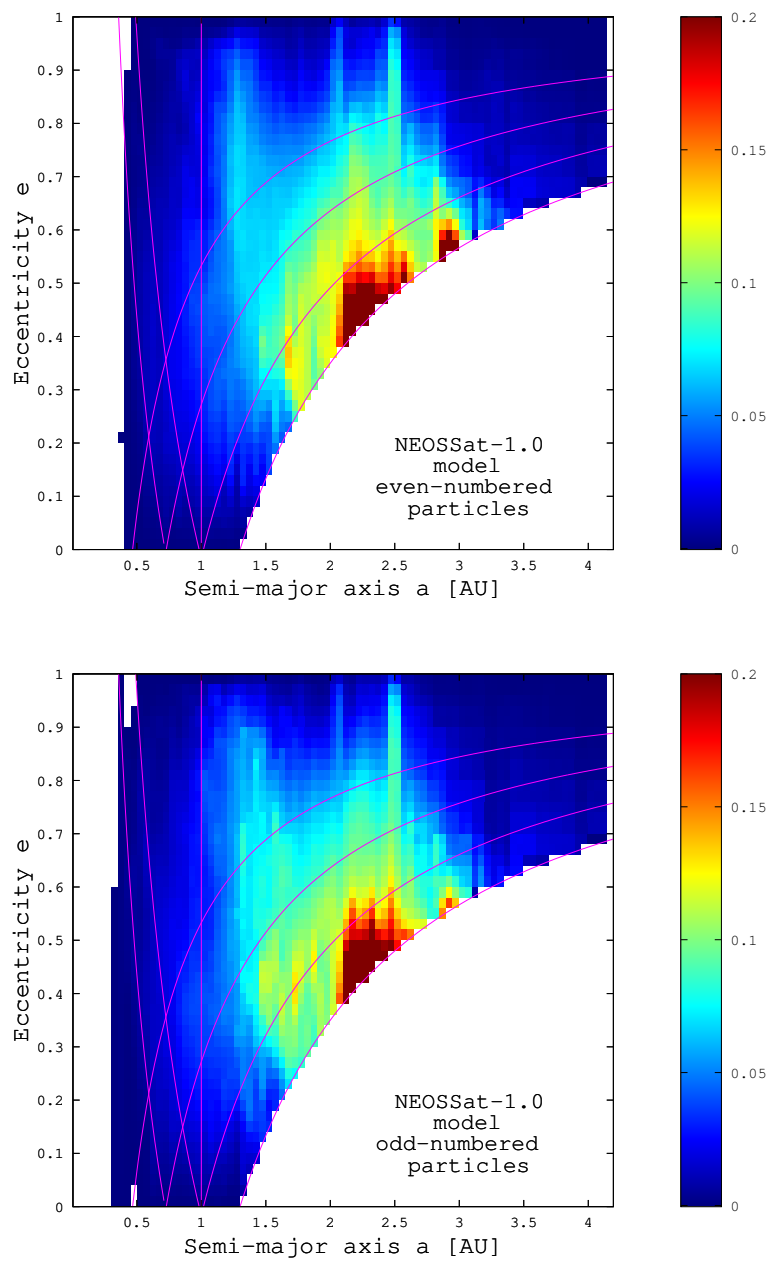

Figure 2.6: Same as Figure 2.3 except computed for the NEOSSat-1.0 model. The discrepancies between these two plots are clearly much smaller than in our B02 recomputation (Figure 2.3). 
$\nu_{6}$ resonance and IMC populations. These two source regions produce similar residence time probability distributions because they partially overlap in phase space. Bottke et al. (2002) recognized this degeneracy and attempted to avoid the problem by integrating a large enough set of test bodies within the clearly defined boundaries of the IMC and $\nu_{6}$ resonance source regions. It is also unclear if the IMC population is correctly bounded and accurately populated in the inital conditions of the integrations. To ensure their bestfit parameters for each source region were as accurate as possible, Bottke et al. (2002) computed the flux rate of material feeding each of the source regions using the number of objects in the steady-state population of each source region and the mean lifetime spent by objects in each source region from their integrations. These flux rates are important in understanding the steady-state populations of both the source regions and the NEOs they supply (Bottke et al., 2002). We accept the method used by Bottke et al. (2002) for computing their weighting factors is sufficient in decreasing the degeneracy problem. To better address the issue, the NEOSSat-1.0 NEO orbital model should be fit to a new NEO survey, such as the NEOWISE data (Mainzer et al., 2011) and the population of the IMC region checked against the refitting. The roughly six times increase in the number of particles integrated in the NEOSSat-1.0 NEO orbital model should combat this degeneracy problem further.

\subsubsection{Gravitational effects only included}

The NEOSSat-1.0 orbital integrations include only point-mass Newtonian gravitational effects. We believe the inclusion of other physical effects will change $\mathcal{R}_{N E O}(a, e, i)$ by less than the current statistical uncertainties.

The $\mathcal{R}_{N E O}(a, e, i)$ computations assume the NEA size distribution does not vary over orbital element space, ignoring the possible effects of collisions, tidal disruptions, or thermal breakup. Collisions (Farinella et al., 1993) and chaotic dissipation (Carruba et al., 2003; Morbidelli \& Nesvorný, 1999) may play a role in the supply mechanisms from the main-belt but are of little importance for km-scale NEAs in the NEO region. The effect of Yarkovsky drift is believed to cause main-belt asteroids to migrate to either larger or smaller $a$, allowing them to drift into the supply resonances (Bottke et al., 2000b; Farinella \& Vokrouhlický, 1999), but again its effect on the large-scale NEO orbital distribution with $q<1.3$ AU should be minor. 


\section{Chapter 3}

\section{Results}

\subsection{New populations discovered within the NEOSSat-1.0 NEO orbital model}

${ }^{11}$ Analysis of the NEOSSat-1.0 orbital model showed the presence of two new rare types of NEAs, which have little or not at all been discussed in the literature.

\subsubsection{Population of objects decoupled from Venus}

Inspection of $\mathcal{R}_{N E O}(a, e, i)$ revealed the existence of NEAs with orbits entirely inside that of Venus. While it is obviously plausible that a Venus encounter could pull an Aten orbit's aphelion below 0.983 AU and thus decouple an NEO from the Earth to become an Atira, a similar process for decoupling from Venus has not been discussed. In the $a<1.0 \mathrm{AU}$ region of $\mathcal{R}_{N E O}(a, e, i)$ (Figure 2.5), there are clear population drop-offs at $Q=0.983 \mathrm{AU}$ (aphelion at Earth) and $Q=0.718 \mathrm{AU}$ (aphelion at Venus). The $0.718<Q<0.983$ AU population are the Atira class NEOs. We have provisionally $^{12}$ named objects with $0.307<Q<0.718$ AU Vatiras, because they are Atiras which are decoupled from Venus. The $Q>0.307$ AU cut is to distinguish Vatiras from the Mercury-decoupled Vulcanoid population, which we define as objects with $Q<0.307$ AU. Figure 2.1 shows the NEO class boundaries we adopt in this paper. The Vatira population is rare and makes up only $\simeq 0.22 \%$ of the steady-state $\mathrm{NEO}$ orbital distribution (Table 2.2).

Most of the particles reaching the Vatira region spend little time in this state integrated over their lifetimes, $\sim 0.25 \mathrm{Myr}$. Particles reach the Vatira region via gravitational scattering in $(a, e, i)$ space due to planetary close

\footnotetext{
${ }^{11} \mathrm{~A}$ version of this chapter has been submitted for publication consideration. Greenstreet, S., Ngo, H., and Gladman, B. (2011) The Orbital Distribution of Near-Earth Objects Inside Earth's Orbit. Icarus.

${ }^{12}$ Provisional because it will be abandoned once the first discovered member of this class will be named.
} 
encounters with Earth, Venus, and Mercury. Objects in the Vatira region typically have $i \simeq 25^{\circ}$ and $e \simeq 0.4$ (Figure 2.5). Only a few examples exist in our integrations of particles long-lived $(>1 \mathrm{Myr})$ in the Vatira region.

The orbital evolution of one long-lived Vatira is shown in Figure 3.1. This particle comes out of the $\nu_{6}$ resonance and rapidly drops to the Vatira state within $\approx 10$ Myr via a set of Earth and Venus encounters; reaching the Vatira state this quickly is rare. About 5 Myr later, it experiences a close encounter which recouples it to Venus. It then repeats this short decouple/recouple evolution before fully decoupling from Venus at around $45 \mathrm{Myr}$ into its lifetime. This decouple/recouple process is dominated by Mercury close encounters. During this evolution, the particle's inclination reaches $\simeq 40^{\circ}$. It then remains in the Vatira state for $\approx 75$ Myr during which time it random walks in $a$ due to planetary close encounters with Mercury before colliding with Mercury at $\approx 125 \mathrm{Myr}$. At termination, $i \simeq 80^{\circ}$ and at terminal impact is at a speed of $\simeq 61 \mathrm{~km} / \mathrm{s}$.

Figure 3.2 shows a second example of a long-lived Vatira. This particle came out of the $\nu_{6}$ resonance and spends most of the first $80 \mathrm{Myr}$ as an Apollo. Plantery close encounters then lower $a$ below that of Venus and it spends $\approx 30$ Myr oscillating between the Aten and Atira classes due to its aphelion oscillating quickly. This rapid oscillation is due to Kozai which occurs when the argument of pericenter librates around either $90^{\circ}$ or $270^{\circ}$ instead of precessing (Kozai, 1962). For orbits with $a<2$ AU, Michel \& Thomas (1996) showed the argument of pericenter can also librate around $180^{\circ}$. By 115 Myr the particle's $a$ drops further due to Mercury encounters and it oscillates between the Vatira and Atira classes. Though the particle shown in Figure 3.2 appears to stay coupled to Venus after $\approx 110 \mathrm{Myr}$ since its aphelion oscillates near $0.718 \mathrm{AU}$, the nodal distances are never outside $0.718 \mathrm{AU}$ for the remainder of the particle integration because Kozai prevents the node from approaching the aphelion distance. It stays alive for another $\simeq 120 \mathrm{Myr}$ due to the high inclinations (between roughly $30^{\circ}$ and $80^{\circ}$ ) reached during its Kozai oscillations, putting it out of harm's way of a planetary collision with Venus, but is pushed into the Sun 228 Myr after leaving the main-belt.

These long-lived Vatiras are rare. With typical lifetimes in the Vatira region of a few hundred thousand years, most NEAs entering the Vatira region do so several times as they experience planetary close encounters which decouple and recouple the particle from Venus before evolving back out in $a$. The median time after injection into the NEA region at which objects first enter the Vatira region is $\approx 17 \mathrm{Myr}$. The median lifetime of objects after their first injection into the Vatira region is $\approx 21 \mathrm{Myr} .42 \%$ of 


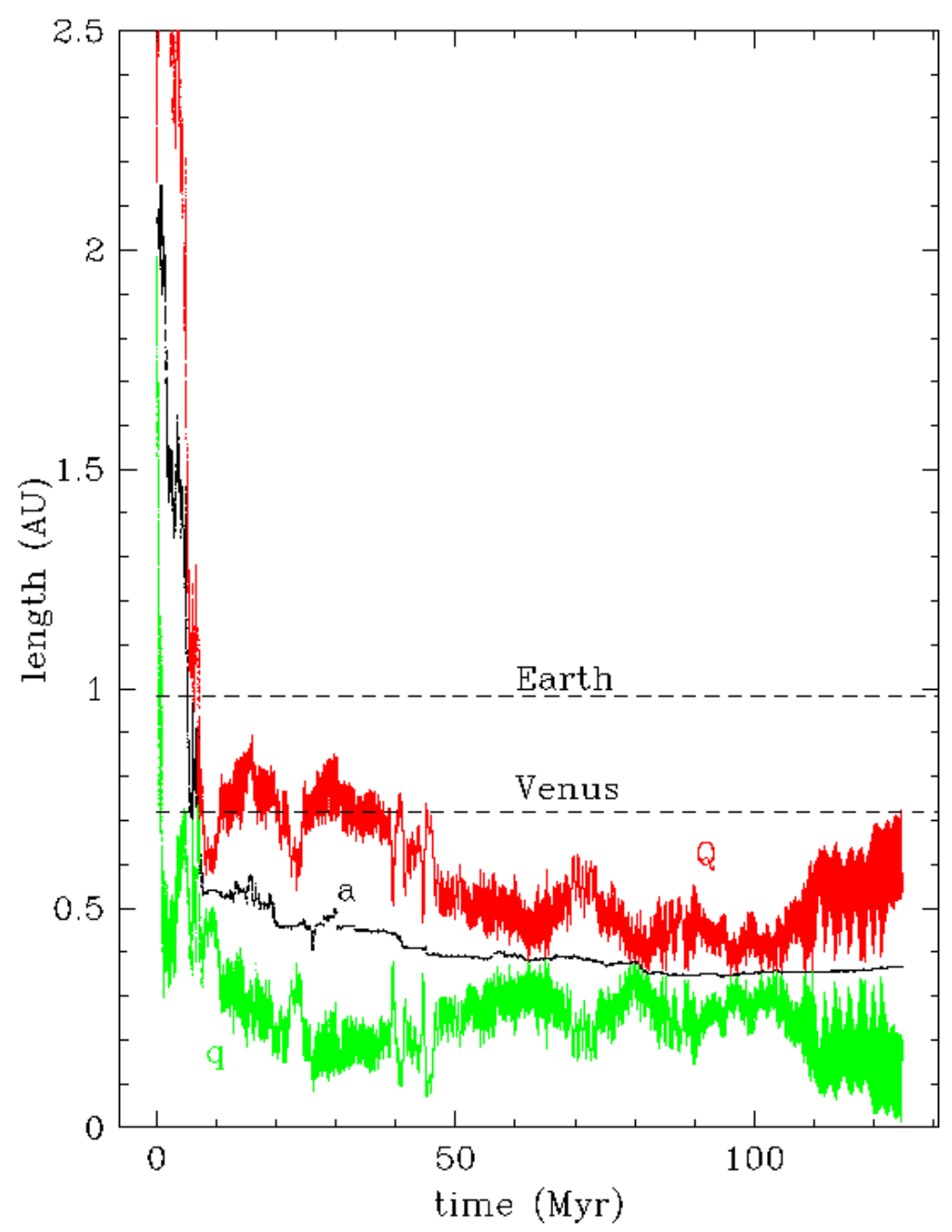

Figure 3.1: This figure shows the aphelion (red), semimajor axis (black), and perihelion (green) versus time of a particle from the NEOSSat-1.0 orbital integrations. This particle rapidly reaches the Vatira state and remains there for the last $\approx 75 \mathrm{Myr}, \approx 60 \%$ of its lifetime. It is a rare occurance for a particle to reach the Vatira state so quickly. This particle strikes Mercury with an impact speed of $\simeq 61 \mathrm{~km} / \mathrm{s}$ at $\simeq 125 \mathrm{Myr}$. 


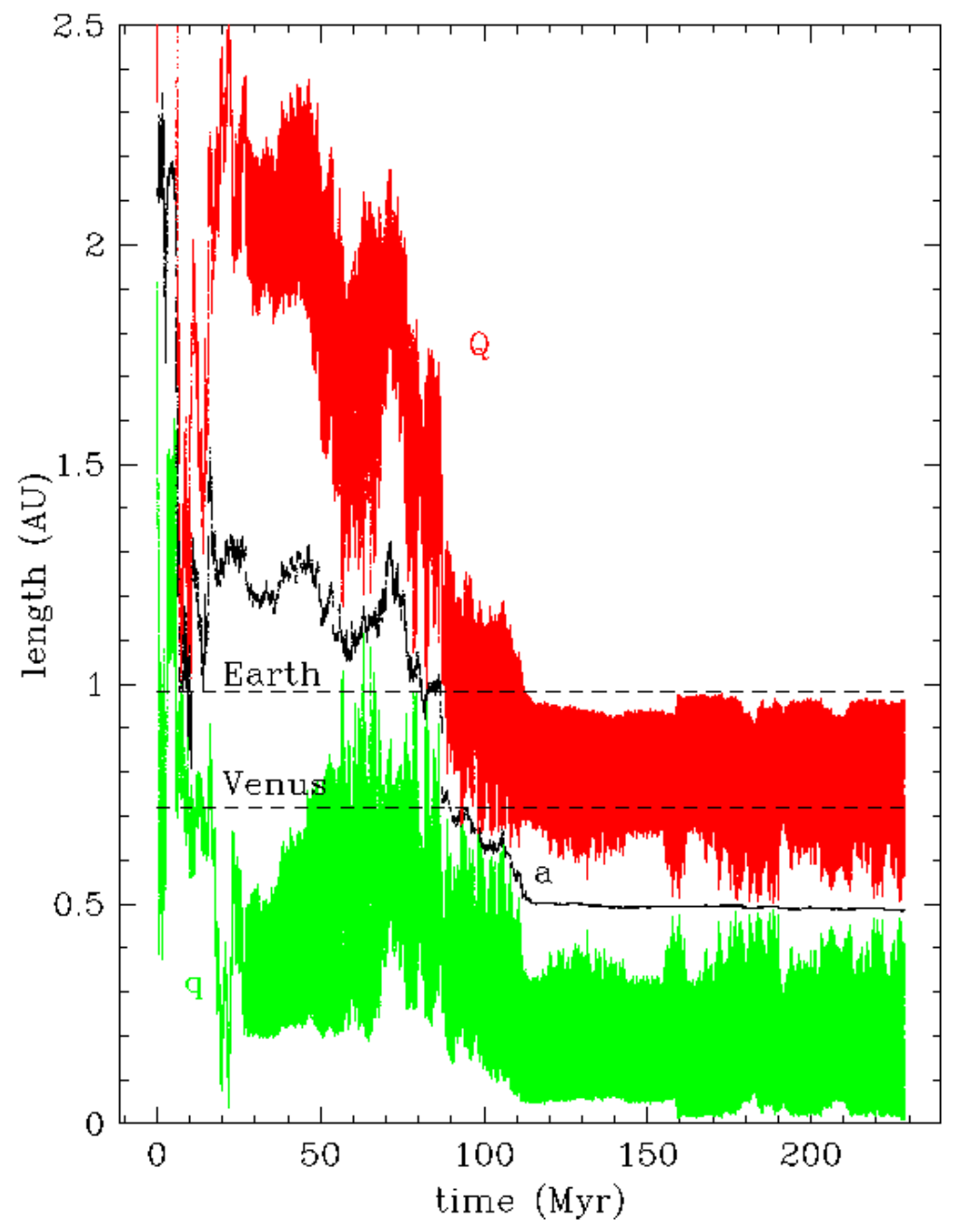

Figure 3.2: Same as Figure 3.1 except of a particle from the NEOSSat1.0 orbital integrations which originates in the $\nu_{6}$ resonance, spending the majority of the first $80 \mathrm{Myr}$ as an Apollo. Close encounters move it to an Aten orbit. Its aphelion then begins oscillating quickly due to Kozai as it fluctuates between the Aten and Atira states. Roughly 30 Myr later, the $a$ drops further due to a planetary close encounter causing it to oscillate between the Atira and Vatira classes. This particle lives long due to the high inclinations (between roughly $30^{\circ}$ and $80^{\circ}$ ) during its Kozai oscillations putting it out of harm's way of a planetary collision with Venus. It becomes a Sun-grazer 228 Myr after leaving the main-belt. 
these objects are terminated because they don't stay decoupled from Venus and eventually collide with Venus. 16\% collide with Earth, $10 \%$ collide with Mercury, and $\simeq 1 \%$ collide with Mars. $20 \%$ evolve onto Sun-grazing orbits, $\simeq 9 \%$ are still alive at the end of the integration, $\simeq 3 \%$ are thrown out of the Solar System, and $\simeq 1 \%$ hit the giant planets. The $\approx 21 \mathrm{Myr}$ time scale is longer than a typical Apollo due to the orbital distance from resonances which can push them onto Sun-grazing orbits (Gladman et al., 2000).

Objects in the Vatira region are nearly unobservable from Earth's surface because they never reach solar elongations $>45^{\circ}$. The Messenger spacecraft could observe them from its position in orbit around Mercury, but given the small-number of these objects that exist, we calculate the probability of Messenger finding a Vatira is extremely small given the aperture and field of view of its imaging system. During rare "eclipse seasons" where Earth's dark limb blocks the Sun, NEOSSat will be able to image this close to the Sun, but again a detection is extremely unlikely. While one may think this population of objects could affect the impact chronology on Mercury, we calculate that only $\simeq 3.2 \%$ of the Mercury impact rate comes from $Q<0.718$ AU orbits (Table 2.3).

\subsubsection{Vulcanoids}

The existence of a non-negligible population of Venus-decoupled Vatiras thus begs the question as to whether any objects reach orbits entirely interior to that of Mercury. Accepted convention would likely to be to call such an object a Vulcanoid, although the term is usually intended to mean an object which has been resident inside Mercury for the entire lifetime of the Solar System (Stern \& Durda, 2000). The most obvious production path would be a Vatira or Atira NEO that suffers a close enounter with Mercury which converts the NEO's orbit to one with aphelion at close to the encounter point. With no planet interior to Mercury, a close encounter cannot be then used to decouple from the planet, but the object could interact with the border of a mean-motion resonance with Mercury (or even high-order resonances of more-distant Venus or Earth) and have its eccentricity reduced. With the Vatira evolutions as example, it is also clear that a large- $i$ Mercury crosser could temporarily decouple as part of a Kozai oscillation.

We examined the NEOSSat-1.0 integrations to search for the appearance of Vulcanoids. Although we found several examples of particles reaching orbits with aphelia $Q$ smaller than Mercury's aphelion, there were no particles logged with $Q<q_{\text {Merc }}=0.307$ AU, which we have adopted as the definition of a Vulcanoid (an orbit entirely inside that of Mercury). The smallest 
observed $Q$ was $Q=0.348 \mathrm{AU}$, and the smallest NEO semimajor axis observed was $a=0.344 \mathrm{AU}$ for the same particle, an orbit entirely inside Mercury's semimajor axis but not perihelion. The fraction of $\mathcal{R}_{N E O}(a, e$, i) with $Q<Q_{\text {Merc }}$ is $0.006 \%$, so we take this as a strong upper limit to the possible value of the Vulcanoid fraction with $Q<0.307$ AU. Given this we would not expect a Vulcanoid from an NEO source to exist larger than $\mathrm{H} \simeq 21$ on average.

If a Mercury-decoupled object is ever found, the possibility should be considered that it is a NEO brought down through the Atira and Vatira regions. The future lifetime of such an object will be limited by Yarkovsky drift (Vokrouhlický et al., 2000) and collisional grinding, but survival of 100-meter scale NEAs for hundreds of Myr is plausible.

\subsubsection{Retrograde NEAs}

Further analysis of the NEOSSat-1.0 integrations uncovered the unexpected production of retrograde orbits from main-belt asteroid sources. We find that NEAs originating in any of the four asteroidal source regions can eventually become retrograde, and typically begin their evolution in the $q<1.3$ AU region random walking in $a$ due to planetary close encounters. The majority of particles which become retrograde (regardless of initial source) do so via the 3:1 mean-motion resonance after they reach it by a random walk in $a$. Once in the resonance, they often experience Kozai oscillations in $e$ and $i$ which can pump their inclinations up to $80^{\circ}$. It is clear that Kozai alone does NOT result in the inclination passing through $90^{\circ}$, because only a tiny fraction (if any) become retrograde outside a resonance even if very high $i$ 's are reached. A dynamical phenomenon in the resonance then causes the inclinations to pass through $90^{\circ}$; the nature of this mechanism is unclear, but it is not planetary close encounters. About $86 \%$ of the retrograde particles stay in the resonance and terminate within 10,000 years when the resonance pushes the high-e particles into the Sun. 98\% of the retrograde NEAs are eventually eliminated from the integrations due to Sun-grazing (Farinella et al., 1994), with only $\simeq 2 \%$ thrown out of the Solar System. Because of their high relative encounter speeds caused by the retrograde orbit, gravitational focussing is negligible and planetary collisions are rare $(<1 \%)$. A minority are kicked out of the retrograde-inducing resonance due to a planetary close encounter, and then can in some cases live tens of millions of years. The details of this mechanism are the subject of another paper; here we provide only the orbital-element distribution of the retrograde NEAs.

Figure 3.3 shows $\mathcal{R}_{180}(a, e, i)$ for the region $a<4.2 \mathrm{AU}, e<1.0$, and 


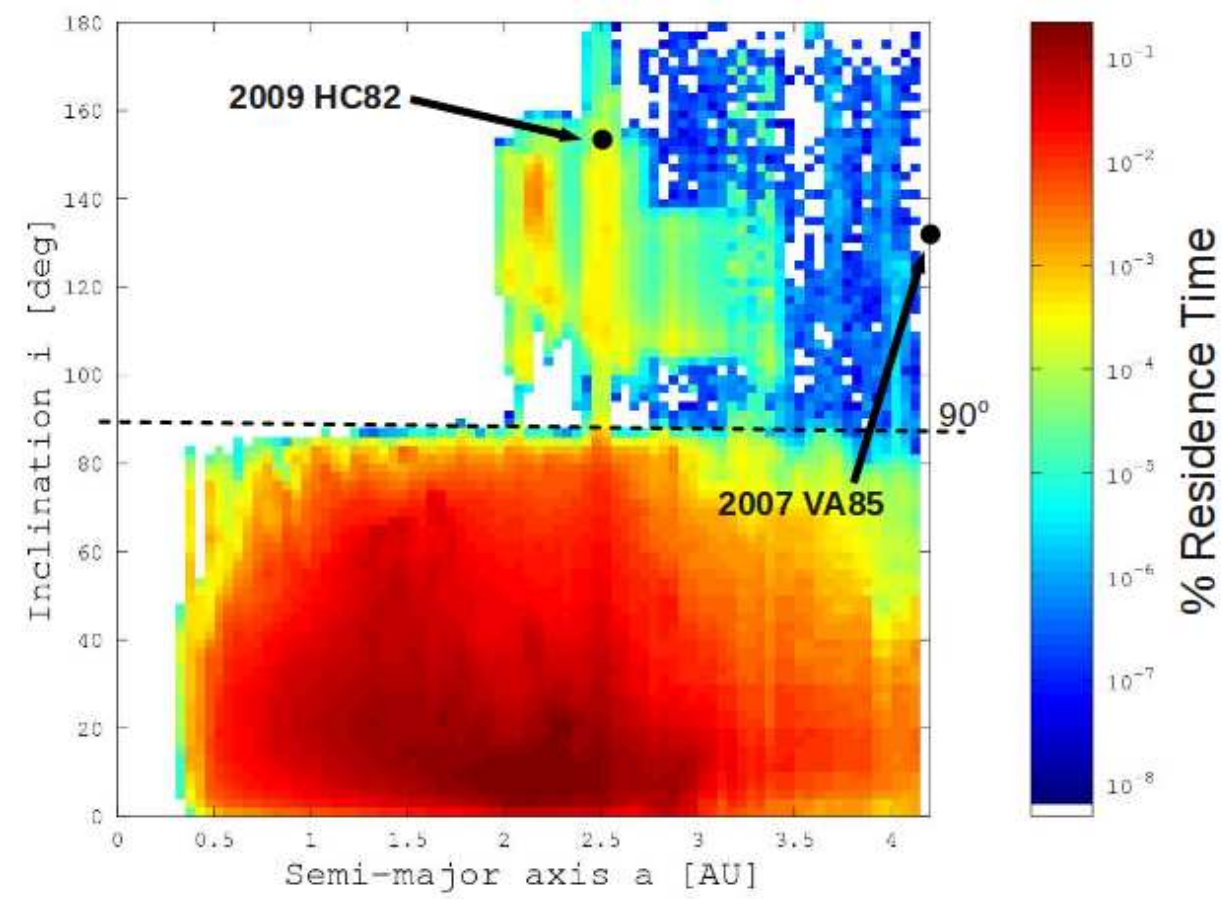

Figure 3.3: Residence time probability distribution, $\mathcal{R}_{N E O}(a, e, i)$, for inclinations up to $180^{\circ}$ for the NEOSSat-1.0 NEO orbital model. The color scheme represents the logarithm of relative density of residence time spent in any given cell in relation to the amount of residence time spent in all cells. The dashed line divides retrograde from direct orbits. The retrograde NEA population makes up $\simeq 0.10 \%$ of the steady-state NEO population. Two known retrograde NEOs are shown. 
$i<180^{\circ}$ (with cell volume $0.05 \mathrm{AU} \times 0.02 \times 2.00^{\circ}$ ). This figure shows the logarithm of the normalized fraction of time spent by particles in each cell. A total of $\simeq 0.10 \%$ of $\mathcal{R}_{180}(a, e, i)$ is in the retrograde NEA population. Although most of the retrograde objects flip while in the $3: 1$ resonance, $\mathcal{R}_{180}(a, e, i)$ shows most of the power for the retrograde objects near $a \simeq 2$ AU. This is due to a single particle which flips in the $3: 1$ resonance early in its lifetime and then spends $\approx 200 \mathrm{Myr}$ near $2 \mathrm{AU}$. There are two known retrograde NEAs: $2007 \operatorname{VA} 85\left(a=4.226 \mathrm{AU}, e=0.736, i=131.769^{\circ}\right)$ and $2009 \operatorname{HC} 82\left(a=2.528 \mathrm{AU}, e=0.807, i=154.519^{\circ}\right)$. These are plotted in Figure 3.3.

\subsection{Impact speeds and rates for Earth, Venus, and Mercury}

Under the steady-state assumption, one can calculate the relative NEO impact rate onto Mercury, Venus, and Earth, as well as the normalized distribution of 'top of the atmosphere' impacts speeds. Our simulations cannot provide this information for Mars due to the existence of $q>1.3$ AU Marscrossing asteroids not modelled here.

This calculation is best performed using the detailed orbital histories of all integrated particles. The intrinsic collision probability and average impact speed onto each of the terrestrial planets were computed by a method described by (Dones et al., 1999; Farinella \& Davis, 1994; Wetherill, 1967). This method gives the expected number of impacts that should be recorded in the simulation, given the particle histories. This was first done separately for each particle in each of the four asteroidal source regions, since different numbers of particles were computed from each source. We find extremely good agreement between the expected number of impacts diagnosed by the collision probability code and the number of impacts directly recorded in the simulations ${ }^{13}$. This agreement gives us confidence in the impact speed distribution simultaneously derived from the collision probability algorithm.

The impact speed distribution from each source region was normalized to the number of particles from that source which entered the $q<1.3$ $\mathrm{AU}$ NEO region. Since each source region is weighted differently in its contribution to the overall NEO population, the resulting normalized impact speed probabilities were weighted by the source region fractions from Bottke

13 For example, from the $\nu_{6}$ source the expected number of impacts for Mercury/Venus/Earth was 106/571/519 while those directly observed in the simulation were 91/563/517; the Poisson errors of the latter are consistent with the former. 
et al. (2002). The resulting distributions were used to create the impact speed distributions for the terrestrial planets shown in Figure 3.4. This figure shows only the speed distribution resulting from asteroidal source regions (neglecting the JFCs).

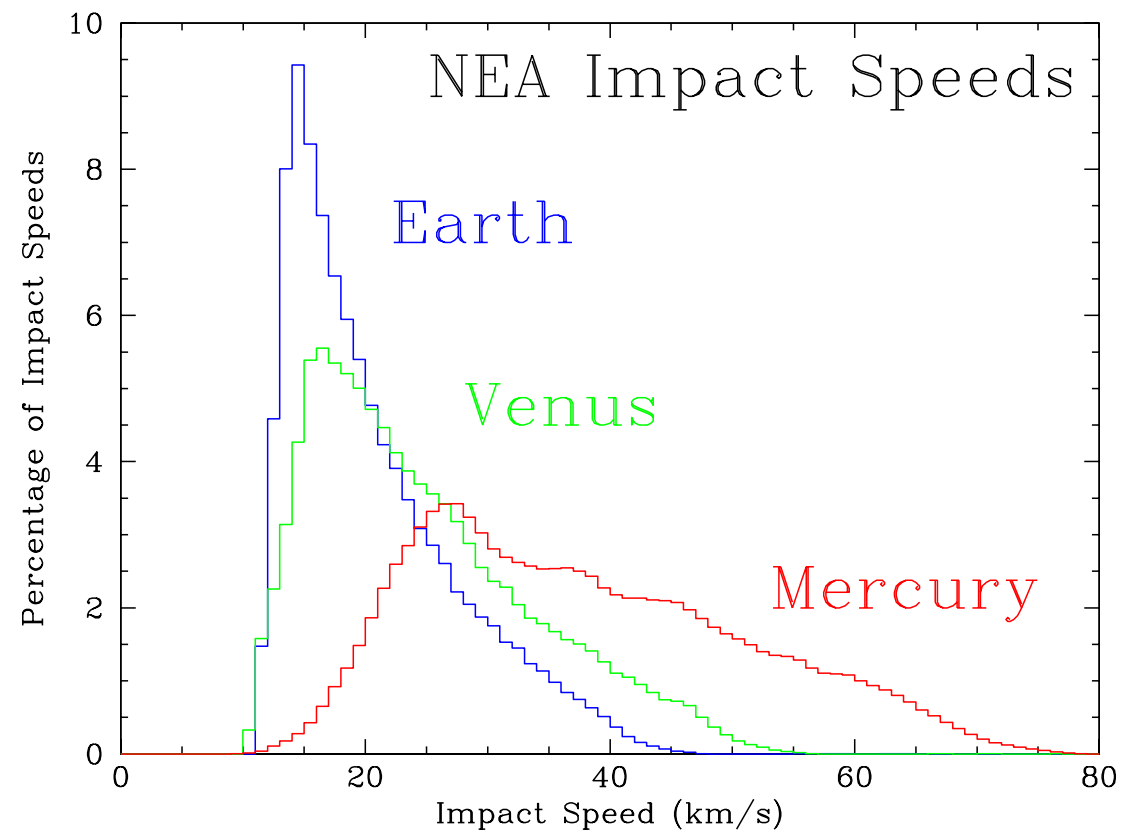

Figure 3.4: 'Top of the atmosphere' impact speed distribution for asteroidal material reaching Earth, Venus, and Mercury. The mean impact speed for Earth is $20.6 \mathrm{~km} / \mathrm{s}$, for Venus is $24.7 \mathrm{~km} / \mathrm{s}$, and for Mercury is $37.9 \mathrm{~km} / \mathrm{s}$. Each planet's distribution is separetely normalized.

As a check, the impact speed distributions were also calculated using the final residence time probability distributions. The orbital elements of each $(a, e, i)$ cell of volume $0.05 \mathrm{AU} \times 0.02 \times 2.00^{\circ}$ were used to determine impact probabilities and mean speed, for the cell center. For each planet, the fractional residence time was multiplied by that cell's collision probability. Since the residence time probability distributions are already normalized, the resulting impact speed distribution could be directly compared to Figure 3.4. Because the residence time distribution has much less detailed information than the particle integrations, the former produces noisier, but otherwise similar, impact speed distributions. This allowed us to compare these distributions with and without the JFC source included in the steady- 
state orbital distribution. Unsurprisingly, because the JFC source is only $\simeq 6 \%$ of the NEO population and the impact probability per JFC is small compared to most NEAs, the impact speed distributions are nearly identical with and without the JFC source included. We thus present only the asteroidal impact speed distribution; workers wishing to include the cometary speed distribution should probably also include all other cometary sources (Halley-type and long-period comets as well).

The NEA impact speed distribution onto Earth is extremely similar to that shown in Gallant et al. (2009) at lower speed resolution, with a strong peak near $15 \mathrm{~km} / \mathrm{sec}$ and long tail out to $45 \mathrm{~km} / \mathrm{sec}$. We use $1 \mathrm{~km} / \mathrm{sec}$ bins and thus have twice the speed resolution of the most recent work (Gallant et al., 2009). The venusian impact speeds (which should be interpreted as 'top of the atmosphere' speeds) peak only slightly faster $(\approx 16 \mathrm{~km} / \mathrm{sec})$, with a tail extending to higher speeds $(55 \mathrm{~km} / \mathrm{s})$ due to the greater heliocentric speeds in closer to the Sun. When converted to cumulative form, the venusian speed distribution is (perhaps amazingly) similar to that approximated by McKinnon et al. (1997). In the case of Venus the interaction of the impactor with the massive atmosphere of course has a critical further role in determining if the surface is reached.

Marchi et al. (2009) published a Mercury impact speed distribution in comparison with the Earth-Moon system based on a fraction of the Bottke et al. (2002) simulations; one must compare our results with their large (L) distribution shown in their Figure 11 which has not been modified from the pure-gravity model. The importance of small-number statistics in the integrations is evident in the impact speed distribution of Marchi et al. (2009); although there is a broad distribution with maximum probability in the $40-50 \mathrm{~km} / \mathrm{sec}$ range, the two narrow peaks (at $\simeq 29$ and $41 \mathrm{~km} / \mathrm{sec}$ ) in their distribution are almost certainly due to two single particles that get lodged in long-lived Mercury-crossing orbits; in comparison, our impact speed distribution for Mercury is much smoother. Recently Le Feuvre \& Wieczorek (2011) produced an improved Mercury impact speed distribution using the complete Bottke et al. (2002) model, which retains the broad maximum at $40-50 \mathrm{~km} / \mathrm{sec}$ but in which these two peaks are absent. In contrast, both of these earlier impact speed distributions lack the strong Mercury impact speed peak we see near $27 \mathrm{~km} / \mathrm{sec}$, that is a robust feature of the NEOSSat-1.0 calcluation, produced by the well-populated 'finger' near $a \simeq 1.2 \mathrm{AU}$ visible in Figure 2.4. The much finer timestep and the presence of Mercury in our integrations means that our $q<0.45$ AU region is more accurately defined; we feel confident in our result, especially because the Earth and Venus speed distributions (less sensitive to the larger timestep) 
agree well with previous estimates. We thus predict a Mercury impact flux more dominated by low-speed impactors than both these workers derived from the Bottke et al. (2002) integrations.

In addition to the impact speed distributions, the NEOSSat-1.0 integrations also permit the computation of relative impact rates on Mercury, Venus, and Earth. While it would be most desirable to have crater formation rates as a function of crater diameter, this depends not only on the speed distribution but also the size distribution of the impactors and the cratering scaling law used. We prefer to remain as close as possible to the direct measurements we can draw from the integrations; our impact speed distributions are available on request for workers who wish to assert their favored impactor size distribution and generate cratering-rate estimates. Instead, we present only the relative 'top of the atmosphere' impact rate, for fixed size impactor. This requires the assumption that the NEO size distribution does not vary in different regions of orbital element space. This is almost certainly untrue for comets, which may split for low perihelia, and may also be false for asteroids. Asteroids on orbits with higher planetary encounter probablility may be prone to tidal disruption (Richardson et al., 1998) potentially altering the size distribution as a large object turns into many smaller ones. However, because comets are a minor fraction of the impact flux and because the rest of the literature also operates under the assumption that the NEA size distribution does not vary over orbital element space, we proceeded to compute impact rates. When we weight the source regions as described above, we find that the impact rate (per NEO per square kilometer of target) ratio is $1.13 \pm 0.01$ for Venus/Earth and $1.21 \pm 0.04$ for Mercury/Earth (Table 3.1). Le Feuvre \& Wieczorek (2008, 2011) found these ratios to be 1.11 and 1.15 respectively. The roughly $5 \%$ higher Mercury impact rate we find would translate into younger surfaces on Mercury when dated via impact-cratering chronology (eg. Marchi et al. (2009)) if rate was the only factor; because we simultaneously also argue that mercurian impact speeds are slightly slower on average, the effect will be reduced.

Note that the various source regions give different planetary impact flux ratios (Table 3.1). Due to their ability to more efficiently deliver long-lived asteroids to high- $e$ orbits, the $\nu_{6}$ and 3:1 provide relatively more Venus and Mercury impactors than the other source regions. If future recalibration of the source fractions $(\alpha)$ occur the planetary impact ratios will also change accordingly.

More interestingly, Table 2.3 listed the fraction of each planet's impact rate which is contributed by NEOs of the various dynamical classes. 
Chapter 3. Results

\begin{tabular}{|l|l|l|l|l|l||l|l|l|}
\hline Impact Ratio & $\nu_{6}$ & $3: 1$ & IMC & OMB & Weighted & Steady & Even & Odd \\
\hline Venus/Earth & 1.22 & 1.19 & 1.10 & 0.77 & 1.13 & $1.12 \pm 0.01$ & 1.11 & 1.12 \\
Mercury/Earth & 1.40 & 1.31 & 1.04 & 0.78 & 1.21 & $1.26 \pm 0.04$ & 1.22 & 1.30 \\
\hline
\end{tabular}

Table 3.1: Impact flux ratios (per unit surface area of target) for Venus/Earth and Mercury/Earth for the individual asteroidal source integrations as well as their weighted average. The asteroidal steady-state ratios (computed from the residence time distribution) and the even/odd splits are given in order to estimate the uncertainties. The quantities measured via the integrations should be more accurate and thus we quote those as best estimates but use the uncertainties from the odd/even splits of $\mathcal{R}_{N E O}(a, e$, i).

Due to geometrical factors and shorter orbital periods, the low- $a$ classes always produce a proportionally larger contribution to each planet's impact flux than their abolute number fraction would imply. For example, Atens make up only $5.0 /(5.0+63.3)=7.4 \%$ of the Earth crossing population, but contribute $20.3 \%$ of the impact flux due to their higher collision probability; this fact is previously discussed in the literature (see, for example Bottke et al. (1994)). We can extend this analyis to even lower semimajor axes. Apollos/Atens/Atiras make up 86.3\%/10.7\%/3.0\% of the Venus-crossing orbits, respectively, but the Atiras contribute $16.5 \%$ of the venusian impact rate. The situation is even more extreme for Mercury, whose Apollo/Aten/Atira/Vatira fractions are 82.3\%/13.2\%/3.4\%/0.8\% of the Mercury-crossing NEOs but for which Apollos make up only $57 \%$ of the impact rate while Vatiras contribute four times their weight, at $3.2 \%$ of the mercurian impact rate. Even still, neglecting the Vatira population would produce only a small error in the mercurian cratering rate but ignoring the Atira-class asteroids, which supply $13 \%$ of the mercurian impact rate, could produce a serious underestimate of the flux. 


\section{Chapter 4}

\section{Future Work}

The NEOSSat-1.0 orbital model was computed to optimize an efficient pointing strategy for NEOSSat to maximize the number of detections, particularly those in the $a<1.0$ AU region. The completion of this model will allow us to perform simulations of the search strategy for NEOSSat as well as its tracking abilities. This can be generalized to any space-based observing platform wishing to observe NEAs, especially those at low solar elongation.

The unexpected discovery of the production of retrograde orbits from asteroidal main-belt sources and the mechanism responsible for flipping orbits through $90^{\circ}$ is another topic to be explored. We have established that most asteroids flip while in the 3:1 mean-motion resonance with Jupiter, however the nature of the mechanism causing the flip in unclear. Since many objects which flip to retrograde orbits experience Kozai oscillations which can bring their inclinations up to $80^{\circ}$, whether Kozai is responsible for further increasing inclinations through $90^{\circ}$ is an interesting question. However, since almost all particles flip while in mean-motion resonances, Kozai alone cannot be the mechanism which puts objects onto retrograde orbits. Whether retrograde orbits are only produced while objects are within mean-motion resonances and not secular resonances is another topic to be explored.

Another future goal is the investigation of tidal effects which can remove small amounts of debris from surfaces of objects which experience very close encounters with Earth and Venus. It is of interest whether the post-encounter orbital distribution of NEOs suffering these close planetary encounters is the same for objects with $a$ just inside and outside Earth's orbit, because the observational color distribution of NEOs appears to be different in these two regions (Binzel et al., 2004).

Once NEOSSat begins providing observational data, provided the necessary circumstances are available, the debiasing of the NEOSSat data using the NEOSSat-1.0 orbital model will be possible.

Refitting the NEOSSat-1.0 orbital model to the NEOWISE data (Mainzer et al., 2011) is also possible now that the model is complete. However, the NEOSSat-1.0 model is not yet pulbic. This will allow further constraint of the best-fit parameters for the NEO source regions. 


\section{Chapter 5}

\section{Summary and Conclusions}

${ }^{14}$ We present the NEOSSat-1.0 orbital model of the steady-state NEO distribution with larger statistics that permit finer resolution and less uncertainty in the $a<1.0$ AU region, the focal point of Canada's NEOSSat's observations. Although this model was produced for the purpose of optimizing the NEOSSat pointing strategy to discover and track NEOs with $a<1.0 \mathrm{AU}$, the model is not dependent upon the satellite in any way. The Atira asteroid class represents $\simeq 1.4 \%$ of the NEO population, and these objects typically have $i \sim 25^{\circ}$ and will thus be found most often at high ecliptic latitudes when viewed from Earth or Earth orbit. Analysis of our integrations provided an accurate characterization of the population of objects decoupled from Venus. This population, which we call Vatiras, constitutes $\simeq 0.22 \%$ of the steady-state NEO orbital distribution. We put an upper bound on the potential Vulcanoids produced via an NEA supply chain. We also show a supply path for the production of retrograde orbits from main-belt asteroid sources. The population of retrogade NEAs is estimated to be $\simeq 0.10 \%$ of the steady-state NEO orbital distribution and is dominantly supplied via the 3:1 mean-motion resonance with Jupiter. We also calculate the relative NEO impact rate onto Mercury, Venus, and Earth, as well as the normalized distribution of impacts speeds and impact flux ratios for Venus/Earth and Mercury/Earth, showing that the Mercury impact rate (at fixed size) is higher and typical impact speeds lower than previous estimates. Upon request, the following products of the NEOSSat-1.0 NEO orbital model are available:

1. The full set of integrations (0.5 terabyte) at 300 year output intervals including the gravitational perturbations of Mercury-Saturn.

2. Residence time probability distribution, $\mathcal{R}(a, e, i)$, for each of the five asteroidal and cometary source regions as well as the combined $\mathcal{R}_{N E O}(a, e, i)$ for $a<4.2 \mathrm{AU}, e<1.0$, and $i<180^{\circ}$.

\footnotetext{
${ }^{14} \mathrm{~A}$ version of this chapter has been submitted for publication consideration. Greenstreet, S., Ngo, H., and Gladman, B. (2011) The Orbital Distribution of Near-Earth Objects Inside Earth's Orbit. Icarus.
} 
3. The fraction in each cell of $\mathcal{R}_{N E O}(a, e, i)$ that came from each source region.

4. 'Top of the atmosphere' asteroidal impact speed distributions for Mercury, Venus, and Earth.

5. A realization of the NEO population consisting of 30,000 NEOs extracted from the NEOSSat-1.0 NEO orbital model. 


\section{Bibliography}

Abell, P. A., Korsmeyer, D. J., Landis, R. R., Jones, T. D., Adamo, D. R., Morrison, D. D., Lemke, L. G., Gonzales, A. A., Gershman, R., Sweetser, T. H., Johnson, L. L., \& Lu, E., 2009, Meteoritics and Planetary Science 44,1825

Binzel, R. P., Morbidelli, A., Merouane, S., DeMeo, F. E., Birlan, M., Vernazza, P., Thomas, C. A., Rivkin, A. S., Bus, S. J., \& Tokunaga, A. T., 2010, Nature 463, 331

Binzel, R. P., Perozzi, E., Rivkin, A. S., Rossi, A., Harris, A. W., Bus, S. J., Valsecchi, G. B., \& Slivan, S. M., 2004, Meteoritics and Planetary Science 39, 351

Bottke, W. F., Jedicke, R., Morbidelli, A., Duncan, M., Petit, J. M., \& Gladman, B., 2000a, Science 288, 2190

Bottke, W. F., Morbidelli, A., Jedicke, R., Petit, J. M., Levison, H. F., Michel, P., \& Metcalfe, T. S., 2002, Icarus 156, 399

Bottke, W. F., Nolan, M., Greenberg, R., \& Kolvoord, R., 1994, in T. Gehrels (ed.), Hazards Due to Comets and Asteroids, pp 337-357, University of Arizona Press

Bottke, W. F., Rubincam, D. P., \& Burns, J. A., 2000b, Icarus 145, 301

Bottke, W. F., Vokrouhlický, D., Broz, M., Nesvorný, D., \& Morbidelli, A., 2001, Science 294, 1693

Carruba, V., Burns, J. A., Bottke, W., \& Nesvorný, D., 2003, Icarus 162, 308

Dones, L., Gladman, B., Melosh, H. J., Tonks, W. B., Levison, H. F., \& Duncan, M., 1999, Icarus 142, 509

Farinella, P. \& Davis, D. R., 1994, Icarus 97, 111 
Farinella, P., Froeschlé, C., Froeschlé, C., Gonczi, R., Hahn, G., Morbidelli, A., \& Valsecchi, G. B., 1994, Nature 371, 315

Farinella, P., Gonczi, R., Froeschlé, C., \& Froeschlé, C., 1993, Icarus 101, 174

Farinella, P. \& Vokrouhlický, D., 1999, Science 283, 1507

Gallant, J., Gladman, B., \& Ćuk, M., 2009, Icarus 202, 371

Gehrels, T. \& Jedicke, R., 1996, Earth, Moon and Planets 72, 233

Gladman, B., Michel, P., Cellino, A., \& Froeschl'e, C., 2000, Icarus 146, 176

Gladman, B. J., Migliorini, F., Morbidelli, A., Zappalà, V., Michel, P., Cellino, A., Froeschlé, C., Levison, H. F., Bailey, M., \& Duncan, M., 1997, Science 277, 197

Granvik, M., Virtanen, J., Oszkiewicz, D., \& Muinonen, K., 2009, Meteoritics and Planetary Science 44, 1853

Greenberg, R. \& Chapman, C. R., 1983, Icarus 55, 455

Greenberg, R. \& Nolan, M. C., 1993, in J. S. Lewis, M. S. Matthews, \& M. L. Guerrieri (eds.), Resources of near-Earth space, pp 473-492, University of Arizona Press

Hildebrand, A. R., Carroll, K. A., Tedesco, E. F., Faber, D. R., Cardinal, R. D., Matthews, J. M., Kuschnig, R., Walker, G. A. H., Gladman, B., Pazder, J., Brown, P. G., Larson, S. M., Worden, S. P., Wallace, B. J., Chodas, P. W., Muinonen, K., \& Cheng, A., 2004, Earth, Moon, and Planets 95, 33

Kozai, Y., 1962, The Astronomical Journal 67, 591

Le Feuvre, M. \& Wieczorek, M. A., 2008, Icarus 197, 291

Le Feuvre, M. \& Wieczorek, M. A., 2011, Icarus 214, 1

Levison, H. F. \& Duncan, M. J., 1994, Icarus 108, 18

Levison, H. F. \& Duncan, M. J., 1997, Icarus 127, 13 
Mainzer, A., Bauer, J., Grav, T., Masiero, J., Cutri, R. M., Dailey, J., Eisenhardt, P., McMillan, R. S., Wright, E., Walker, R., Jedicke, R., Spahr, T., Tholen, D., Alles, R., Beck, R., Brandenburg, H., Conrow, T., Evan, T., Fowler, J., Jarrett, T., Marsh, K., Masci, F., McCallon, H., Wheelock, S., Wittman, M., Wyatt, P., DeBaun, E., Elliott, G., Elsbury, D., Gautier, T., I., Gomillion, S., Leisawitz, D., Maleszewski, C., Micheli, M., \& Wilkins, A., 2011, The Astrophysical Journal 731, 4936

Marchi, S., Mottola, S., Cremonese, G., Massironi, M., \& Martellato, E., 2009, The Astronomical Journal 137

McKinnon, W. B., Zahnle, K. J., Ivanov, B. A., \& Melosh, H. J., 1997, in S. W. Bougher, D. M. Hunten, \& R. J. Phillips (eds.), Venus II, pp 969-1014, University of Arizona Press

Michel, P. \& Froeschlé, C., 1997, Icarus 128, 230

Michel, P. \& Thomas, F., 1996, Astronomy and Astrophysics 307, 310

Michel, P., Zappalà, V., Cellino, A., \& Tanga, P., 2000, Icarus 143, 421

Morbidelli, A. \& Nesvorný, D., 1999, Icarus 139, 295

Richardson, D. C., Bottke, W. F., \& Love, S. G., 1998, Icarus 134, 47

Sarty, G. E., Szalai, T., Kiss, L. L., Matthews, J. M., Wu, K., Kuschnig, R., Guenther, D. B., Moffat, A. F. J., Rucinski, S. M., Sasselov, D., Weiss, W. W., Huziak, R., Johnston, H. M., Phillips, A., \& Ashley, M. C. B., 2011, Monthly Notices of the Royal Astronomical Society 411, 1293

Schmadel, L. D., 2009, Dictionary of Minor Planet Names: Addendum to 5th Edition: 2006-2008, Springer-Verlag Berlin Heidelberg

Stern, S. A. \& Durda, D. D., 2000, Icarus 143, 360

Stuart, J. S., 2001, Science 294, 1691

Veverka, J., Farquhar, B., Robinson, M., Thomas, P., Murchie, S., Harch, A., Antreasian, P. G., Chesley, S. R., Miller, J. K., Owen, W. M., Williams, B. G., Yeomans, D., Dunham, D., Heyler, G., Holdridge, M., Nelson, R. L., Whittenburg, K. E., Ray, J. C., Carcich, B., Cheng, A., Chapman, C., Bell, J. F., Bell, M., Bussey, B., Clark, B., Domingue, D., Gaffey, M. J., Hawkins, E., Izenberg, N., Joseph, J., Kirk, R., Lucey, P., Malin, M., McFadden, L., Merline, W. J., Peterson, C., Prockter, L., Warren, J., \& Wellnitz, D., 2001, Nature 413, 390 
Vokrouhlický, D., Farinella, P., \& Bottke, W. F., 2000, Icarus 148, 147

Walker, G., Matthews, J., Kuschnig, R., Johnson, R., Rucinski, S., Pazder, J., Burley, G., Walker, A., Skaret, K., Zee, R., Grocott, S., Carroll, K., Sinclair, P., Sturgeon, D., \& Harron, J., 2003, The Publications of the Astronomical Society of the Pacific 115, 1023

Wetherill, G. W., 1967, Journal of Geophysical Research 72, 2429

Wetherill, G. W., 1979, Icarus 37, 96

Wetherill, G. W. \& Faulkner, J., 1981, Icarus 46, 390

Wisdom, J., 1985, Nature 315, 731

Yano, H., Kubota, T., Miyamoto, H., Okada, T., Scheeres, D., Takagi, Y., Yoshida, K., Abe, M., Abe, S., Barnouin-Jha, O., Fujiwara, A., Hasegawa, S., Hashimoto, T.and Ishiguro, M., Kato, M., Kawaguchi, J., Mukai, T., Saito, J., Sasaki, S., \& Yoshikawa, M., 2006, Science 312, 1350 


\section{Appendix A}

\section{Supplementary Information}

The residence time probability distribution, $\mathcal{R}(a, e, i)$, for each of the five source regions is provided here. Each source region is combined via Equation 2.1 to produce the master residence time distribution, $\mathcal{R}_{N E O}(a, e, i)$, shown in Figure 2.4.
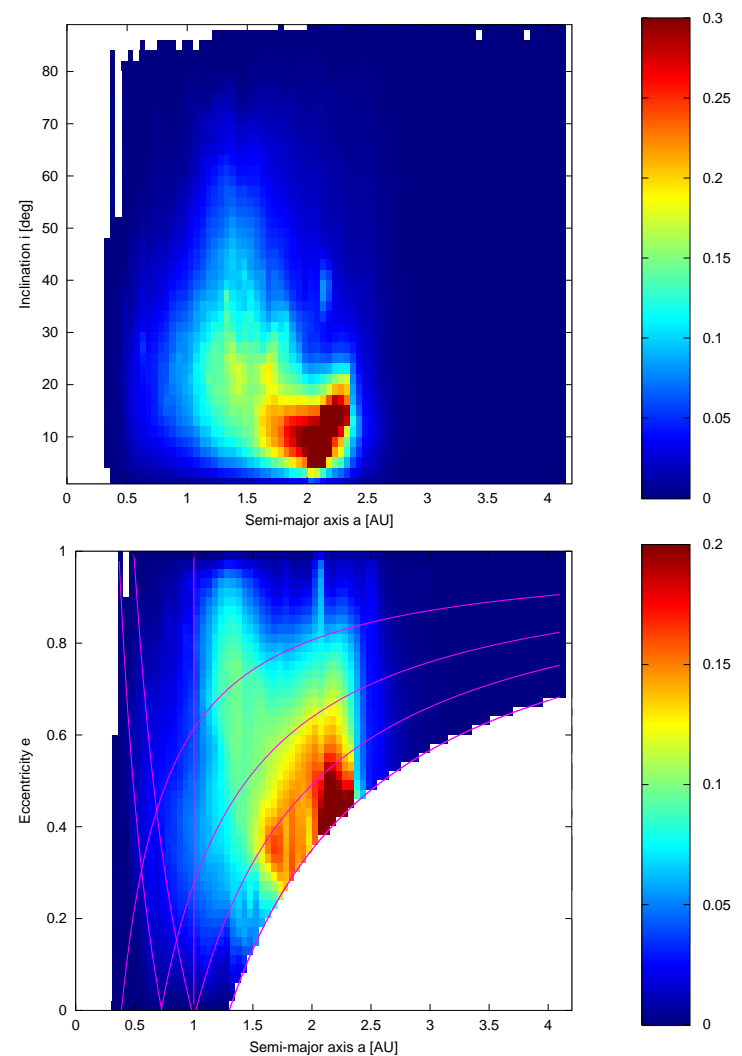

Figure A.1: Residence time probability distribution, $\mathcal{R}_{\nu_{6}}(a, e, i)$, for the NEOSSat-1.0 NEO orbital model. This figure is constructed the same as Figure 2.4 except it only displays the $\nu_{6}$ source. 

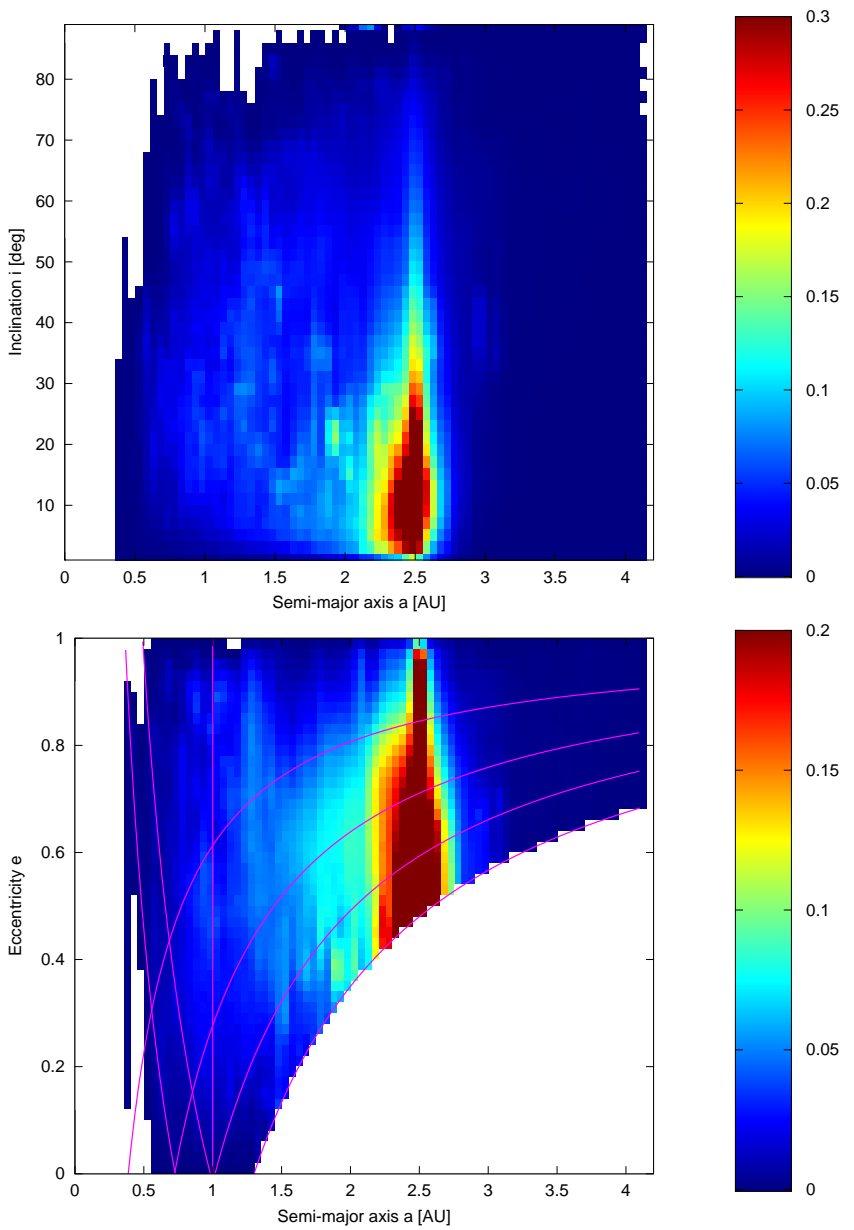

Figure A.2: Residence time probability distribution, $\mathcal{R}_{3: 1}(a, e, i)$, for the NEOSSat-1.0 NEO orbital model. This figure is constructed the same as Figure 2.4 except it only displays the $3: 1$ source. 

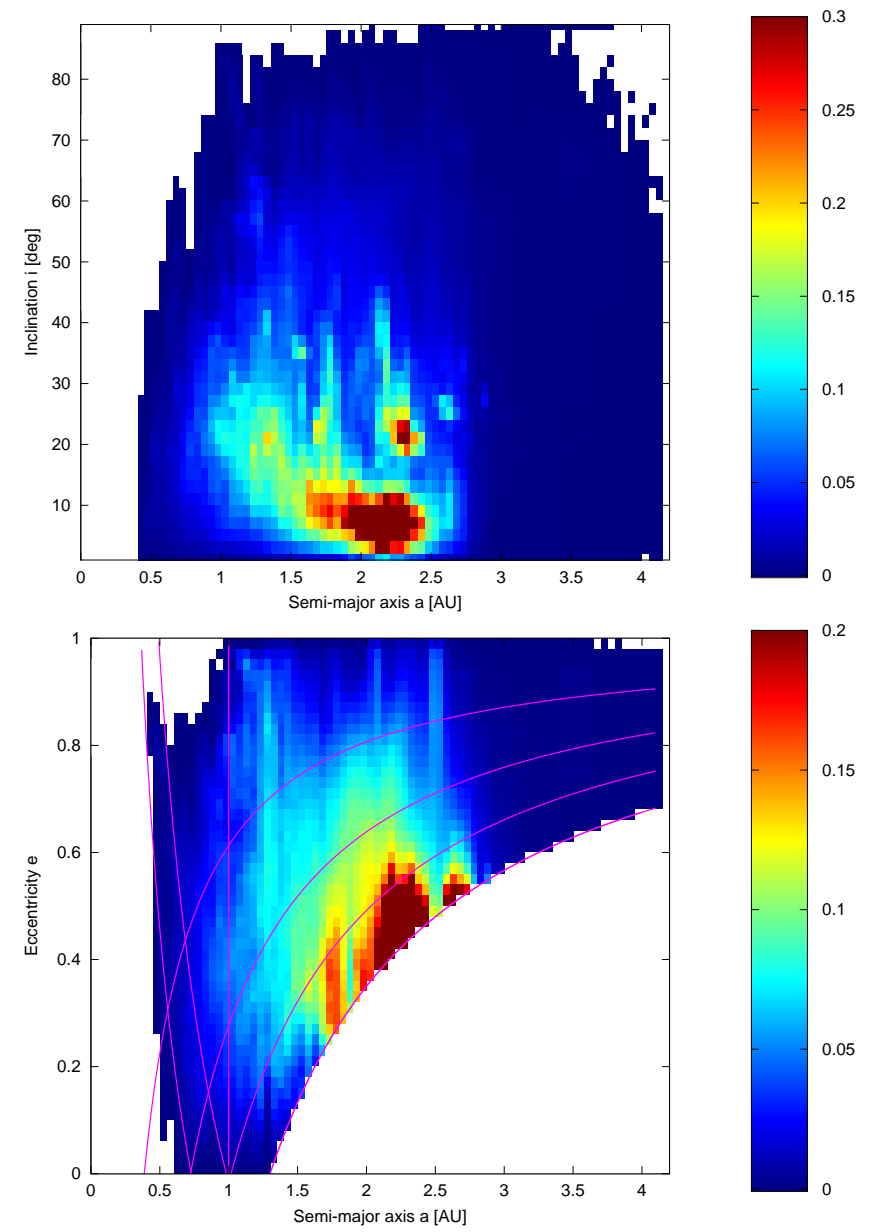

Figure A.3: Residence time probability distribution, $\mathcal{R}_{I M C}(a, e, i)$, for the NEOSSat-1.0 NEO orbital model. This figure is constructed the same as Figure 2.4 except it only displays the IMC source. 

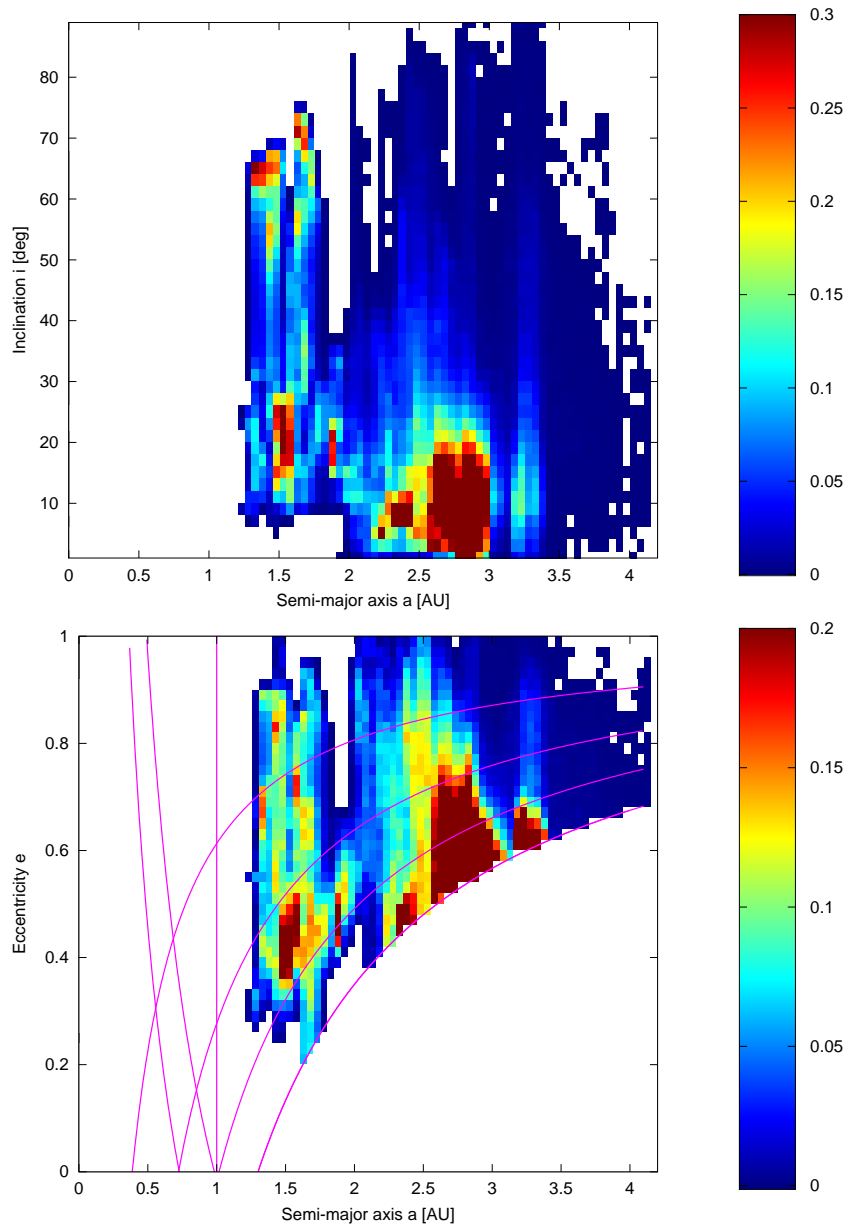

Figure A.4: Residence time probability distribution, $\mathcal{R}_{O M B}(a, e, i)$, for the NEOSSat-1.0 NEO orbital model. This figure is constructed the same as Figure 2.4 except it only displays the OMB source. 

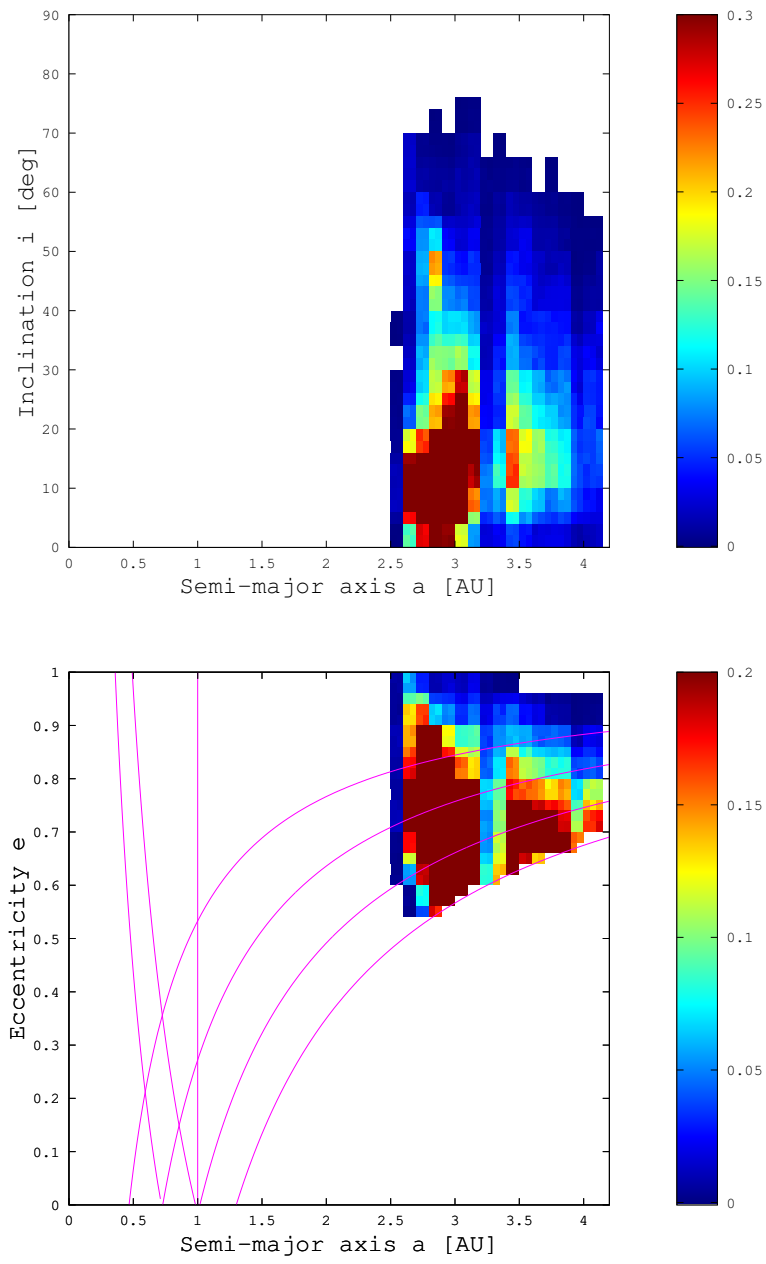

Figure A.5: Residence time probability distribution, $\mathcal{R}_{J F C}(a, e, i)$, for the NEOSSat-1.0 NEO orbital model. This figure is constructed the same as Figure 2.4 except it only displays the JFC source. 


\section{Appendix B}

\section{Additional Notes on Co-Authorship}

This appendix contians additional information concerning the specific contributions made by Sarah Greenstreet to the various components of the analysis of the NEOSSat-1.0 orbital model. Analysis of the numerical integrations dominantly inherited by Sarah Greenstreet was done using pieces of code which monitor the orbital evolution histories of the integrated test particles, construct the residence time distributions of test particles, estimate uncertainties in the residence time distributions, compute the impact speed distributions for Mercury, Venus, and Earth as well as the collision probability distribution for the terrestrial planets. Sarah Greenstreet contributed the following data analysis and scientific interpretation of the NEOSSat-1.0 NEO orbital integrations to the construction of the steady-state NEO orbital distribution model discussed in this thesis.

- Orbital evolution histories: The analysis of the Vatira population:

- computation of the smallest aphelion distance of each test particle

- extraction of orbital histories of particles upon becoming Vatiras

the retrograde NEA population:

- computation of the semimajor axes particles have at the instant they become retrograde

- extraction of orbital histories of particles upon becoming retrograde

and particle orbital evolutions:

- extraction of the median inclination trend from 4.2 AU to Sun

- computation of the region of $(a, e, i)$ space contributing to the dominant features in the impact speed distribution 
was done by Sarah Greenstreet with suggestions made by Dr. Gladman.

- Residence time distributions, $\mathcal{R}_{N E O}(a, e, i)$ :

- expanding the residence time distributions for $a<4.2 \mathrm{AU}, e<$ 1.0 , and $i<90^{\circ}$ to $a<4.2 \mathrm{AU}, e<1.0$, and $i<180^{\circ}$

- computaion of the fraction of $\mathcal{R}_{N E O}(a, e, i)$ from each source region for each NEO class

- rebinning $\mathcal{R}_{J F C}(a, e, i)$ used by (Bottke et al., 2002) to match our cell volume $\left(0.05 \mathrm{AU} \times 0.02 \times 2.00^{\circ}\right)$

- and the source region mapper code

were all written by Sarah Greenstreet.

- Uncertianty estimates for residence time distributions:

- computation of $\mathcal{R}_{N E O}(a, e, i)$ for even- and odd-numbered particle contributions

was done by Sarah Greenstreet with suggestions made by Dr. Gladman.

- Collision probability code: This code computes the collision probability distribution for any planet as well as the impact speed distribution.

- modification of the collision probability code to run on the residence time distributions in addition to the numerical integration particle histories

- expansion of the collision probability code to run on each individual source region

- additional code to compute the planet crossing impact rate contributions from each NEO class (Table 2.3)

- the computation of the impact flux ratios (per unit surface area of target) for Venus/Earth and Mercury/Earth for:

* the individual asteroidal source integrations

* the weighted average of the impact flux ratios for the individual asteroidal source integrations

* the asteroidal steady-state ratios (computed from the residence time distribution) 
* and the even/odd particle splits in order to estimate uncertainties

was done by Sarah Greenstreet with suggestions made by Dr. Gladman.

- Additional analysis:

- a realization of the NEO population consisting of 30,000 NEOs extracted from the NEOSSat-1.0 NEO orbital model

was computed by Sarah Greenstreet with suggestions made by Dr. Gladman.

The figures as they are shown in this thesis were constructed by Sarah Greenstreet except for Figure 1.1, which was made by Henry Ngo, and Figure 2.1, which was made by Dr. Gladman. 